\title{
A novel approach to neutrosophic sets in UP-algebras
}

\author{
Metawee Songsaeng, Aiyared lampan* \\ Department of Mathematics, School of Science, University of Phayao, Phayao 56000, Thailand.
}

\begin{abstract}
The notion of neutrosophic sets in UP-algebras was introduced by Songsaeng and Iampan [M. Songsaeng, A. Iampan, Eur. J. Pure Appl. Math., 12 (2019), 1382-1409]. In this paper, the notions of special neutrosophic UP-subalgebras, special neutrosophic near UP-filters, special neutrosophic UP-filters, special neutrosophic UP-ideals, and special neutrosophic strong UP-ideals of UP-algebras are introduced, and several properties are investigated. Conditions for neutrosophic sets to be special neutrosophic UP-subalgebras, special neutrosophic near UP-filters, special neutrosophic UP-filters, special neutrosophic UP-ideals, and special neutrosophic strong UP-ideals of UP-algebras are provided. Relations between special neutrosophic UP-subalgebras (resp., special neutrosophic near UP-filters, special neutrosophic UP-filters, special neutrosophic UP-ideals, special neutrosophic strong UP-ideals) and their level subsets are considered.
\end{abstract}

Keywords: UP-algebra, special neutrosophic UP-subalgebra, special neutrosophic near UP-filter, special neutrosophic UP-filter, special neutrosophic UP-ideal, special neutrosophic strong UP-ideal.

2020 MSC: 03G25, 03B52, 03B60.

(C)2020 All rights reserved.

\section{Introduction and preliminaries}

Smarandache [19] introduced the notion of neutrosophic sets in 1999 which is a more general platform that extends the notions of classic sets, (intuitionistic) fuzzy sets and interval valued (intuitionistic) fuzzy sets (see $[19,20])$. Neutrosophic set theory is applied to various part which is referred to the site

\section{http:/ / fs.gallup.unm.edu/neutrosophy.htm}

The above-mentioned part has been derived from [25]. Wang et al. [28] introduced the notion of interval neutrosophic sets in 2005. Khan et al. [13] introduced the notion of neutrosophic $\mathcal{N}$-structures and their applications in semigroups in 2017. Jun et al. [8,9] studied neutrosophic $\mathcal{N}$-structures to BCK/BCIalgebras and neutrosophic subalgebras of several types in BCK/BCI-algebras in 2017. Jun et al. [10] studied neutrosophic positive implicative $\mathcal{N}$-ideals in BCK-algebras in 2018. Kim et al. [14] studied generalizations of neutrosophic subalgebras in BCK/BCI-algebras based on neutrosophic points in 2018.

\footnotetext{
*Corresponding author

Email addresses: metawee.faith@gmail.com (Metawee Songsaeng), aiyared.ia@up.ac.th (Aiyared Iampan)

doi: $10.22436 /$ jmcs.021.01.08
}

Received: 2019-11-04 Revised: 2020-01-21 Accepted: 2020-02-10 
Songsaeng and Iampan [23] introduced the notions of neutrosophic UP-subalgebras, neutrosophic near UP-filters, neutrosophic UP-filters, neutrosophic UP-ideals, and neutrosophic strong UP-ideals of UPalgebras in UP-algebras.

In this paper, the notions of special neutrosophic UP-subalgebras, special neutrosophic near UP-filters, special neutrosophic UP-filters, special neutrosophic UP-ideals, and special neutrosophic strong UP-ideals of UP-algebras are introduced, and several properties are investigated. Conditions for neutrosophic sets to be special neutrosophic UP-subalgebras, special neutrosophic near UP-filters, special neutrosophic UP-filters, special neutrosophic UP-ideals, and special neutrosophic strong UP-ideals of UP-algebras are provided. Relations between special neutrosophic UP-subalgebras (resp., special neutrosophic near UPfilters, special neutrosophic UP-filters, special neutrosophic UP-ideals, special neutrosophic strong UPideals) and their level subsets are considered.

Before we begin our study, we will give the definition and useful properties of UP-algebras.

An algebra $X=(X, \cdot, 0)$ of type $(2,0)$ is called a UP-algebra, where $X$ is a nonempty set, $\cdot$ is a binary operation on $X$, and 0 is a fixed element of $X$ (i.e., a nullary operation) if it satisfies the following axioms:

$(\mathrm{UP}-1)(\forall x, y, z \in X)((y \cdot z) \cdot((x \cdot y) \cdot(x \cdot z))=0)$

(UP-2) $(\forall x \in X)(0 \cdot x=x)$;

(UP-3) $(\forall x \in X)(x \cdot 0=0)$; and

$(\mathrm{UP}-4)(\forall x, y \in X)(x \cdot y=0, y \cdot x=0 \Rightarrow x=y)$.

The branch of the logical algebra, UP-algebras were introduced by Iampan [5], and he proved that the notion of UP-algebras is a generalization of KU-algebras (see [16]). Later Somjanta et al. [21] studied fuzzy UP-subalgebras, fuzzy UP-ideals and fuzzy UP-filters of UP-algebras. Guntasow et al. [4] studied fuzzy translations of a fuzzy set in UP-algebras. Kesorn et al. [12] studied intuitionistic fuzzy sets in UP-algebras. Kaijae et al. [11] studied anti-fuzzy UP-ideals and anti-fuzzy UP-subalgebras. Tanamoon et al. [26] studied Q-fuzzy sets in UP-algebras. Sripaeng et al. [24] studied anti Q-fuzzy UP-ideals and anti Q-fuzzy UP-subalgebras of UP-algebras. Dokkhamdang et al. [3] studied Generalized fuzzy sets in UP-algebras. Songsaeng and Iampan [22] studied $\mathcal{N}$-fuzzy UP-algebras and its level subsets, etc.

Example 1.1 ([18]). Let $X$ be a universal set and let $\Omega \in \mathcal{P}(X)$, where $\mathcal{P}(X)$ means the power set of $X$. Let $\mathcal{P}_{\Omega}(X)=\{A \in \mathcal{P}(X) \mid \Omega \subseteq A\}$. Define a binary operation $\cdot$ on $\mathcal{P}_{\Omega}(X)$ by putting $A \cdot B=B \cap\left(A^{C} \cup \Omega\right)$ for all $A, B \in \mathcal{P}_{\Omega}(X)$, where $A{ }^{C}$ means the complement of a subset $A$. Then $\left(\mathcal{P}_{\Omega}(X), \cdot, \Omega\right)$ is a UP-algebra and we shall call it the generalized power UP-algebra of type 1 with respect to $\Omega$. Let $\mathcal{P}^{\Omega}(X)=\{A \in \mathcal{P}(X) \mid A \subseteq \Omega\}$. Define a binary operation $*$ on $\mathcal{P}^{\Omega}(X)$ by putting $A * B=B \cup\left(A^{C} \cap \Omega\right)$ for all $A, B \in \mathcal{P}^{\Omega}(X)$. Then $\left(\mathcal{P}^{\Omega}(X), *, \Omega\right)$ is a UP-algebra and we shall call it the generalized power UP-algebra of type 2 with respect to $\Omega$. In particular, $(\mathcal{P}(X), \cdot, \emptyset)$ is a UP-algebra and we shall call it the power UP-algebra of type 1 , and $(\mathcal{P}(X), *, X)$ is a UP-algebra and we shall call it the power UP-algebra of type 2.

Example 1.2 ([3]). Let $\mathbb{N}$ be the set of all natural numbers with two binary operations $\circ$ and $\bullet$ defined by

$$
(\forall x, y \in \mathbb{N})\left(x \circ y=\left\{\begin{array}{ll}
y, & \text { if } x<y, \\
0, & \text { otherwise, }
\end{array}\right)\right.
$$

and

$$
(\forall x, y \in \mathbb{N})\left(x \bullet y=\left\{\begin{array}{ll}
y, & \text { if } x>y \text { or } x=0, \\
0, & \text { otherwise, }
\end{array}\right) .\right.
$$

Then $(\mathbb{N}, \circ, 0)$ and $(\mathbb{N}, \bullet, 0)$ are UP-algebras. 
Example 1.3 ([15]). Let $X=\{0,1,2,3,4,5\}$ be a set with a binary operation - defined by the following Cayley table:

\begin{tabular}{c|llllll}
$\cdot$ & 0 & 1 & 2 & 3 & 4 & 5 \\
\hline 0 & 0 & 1 & 2 & 3 & 4 & 5 \\
1 & 0 & 0 & 2 & 3 & 2 & 5 \\
2 & 0 & 1 & 0 & 3 & 1 & 5 \\
3 & 0 & 1 & 2 & 0 & 4 & 5 \\
4 & 0 & 0 & 0 & 3 & 0 & 5 \\
5 & 0 & 0 & 2 & 0 & 2 & 0
\end{tabular}

Then $(X, \cdot, 0)$ is a UP-algebra.

For more examples of UP-algebras, see [1, 2, 6, 17, 18].

In a UP-algebra $X=(X, \cdot, 0)$, the following assertions are valid (see $[5,6])$.

$$
\begin{aligned}
& (\forall x \in X)(x \cdot x=0) \\
& (\forall x, y, z \in X)(x \cdot y=0, y \cdot z=0 \Rightarrow x \cdot z=0), \\
& (\forall x, y, z \in X)(x \cdot y=0 \Rightarrow(z \cdot x) \cdot(z \cdot y)=0), \\
& (\forall x, y, z \in X)(x \cdot y=0 \Rightarrow(y \cdot z) \cdot(x \cdot z)=0), \\
& (\forall x, y \in X)(x \cdot(y \cdot x)=0) \\
& (\forall x, y \in X)((y \cdot x) \cdot x=0 \Leftrightarrow x=y \cdot x) \\
& (\forall x, y \in X)(x \cdot(y \cdot y)=0), \\
& (\forall a, x, y, z \in X)((x \cdot(y \cdot z)) \cdot(x \cdot((a \cdot y) \cdot(a \cdot z)))=0), \\
& (\forall a, x, y, z \in X)((((a \cdot x) \cdot(a \cdot y)) \cdot z) \cdot((x \cdot y) \cdot z)=0), \\
& (\forall x, y, z \in X)(((x \cdot y) \cdot z) \cdot(y \cdot z)=0), \\
& (\forall x, y, z \in X)(x \cdot y=0 \Rightarrow x \cdot(z \cdot y)=0), \\
& (\forall x, y, z \in X)(((x \cdot y) \cdot z) \cdot(x \cdot(y \cdot z))=0), \text { and } \\
& (\forall a, x, y, z \in X)(((x \cdot y) \cdot z) \cdot(y \cdot(a \cdot z))=0) .
\end{aligned}
$$

From [5], the binary relation $\leqslant$ on a UP-algebra $X=(X, \cdot, 0)$ defined as follows:

$$
(\forall x, y \in X)(x \leqslant y \Leftrightarrow x \cdot y=0) .
$$

Definition $1.4([4,5,7,21])$. A nonempty subset $S$ of a UP-algebra $(X, \cdot, 0)$ is called

(1) a UP-subalgebra of $X$ if $(\forall x, y \in S)(x \cdot y \in S)$;

(2) a near UP-filter of $X$ if

(i) the constant 0 of $X$ is in $S$, and

(ii) $(\forall x, y \in X)(y \in S \Rightarrow x \cdot y \in S)$;

(3) a UP-filter of $X$ if

(i) the constant 0 of $X$ is in $S$, and

(ii) $(\forall x, y \in X)(x \cdot y \in S, x \in S \Rightarrow y \in S)$;

(4) a UP-ideal of $X$ if

(i) the constant 0 of $X$ is in $S$, and

(ii) $(\forall x, y, z \in X)(x \cdot(y \cdot z) \in S, y \in S \Rightarrow x \cdot z \in S)$; 
(5) a strong UP-ideal (renamed from a strongly UP-ideal) of $X$ if

(i) the constant 0 of $X$ is in $S$, and

(ii) $(\forall x, y, z \in X)((z \cdot y) \cdot(z \cdot x) \in S, y \in S \Rightarrow x \in S)$.

Guntasow et al. [4] and Iampan [7] proved that the notion of UP-subalgebras is a generalization of near UP-filters, near UP-filters is a generalization of UP-filters, UP-filters is a generalization of UP-ideals, and UP-ideals is a generalization of strong UP-ideals. Moreover, they also proved that the only strong UP-ideal of a UP-algebra $X$ is $X$.

\section{NSs of special types in UP-algebras}

The notion of a fuzzy set in a nonempty set was first considered by Zadeh [29] in 1965. A fuzzy set (briefly, FS) in a nonempty set $X$ (or a fuzzy subset of $X$ ) is an arbitrary function $f: X \rightarrow[0,1]$, where $[0,1]$ is the unit segment of the real line, and the fuzzy set $\bar{f}$ defined by $\bar{f}(x)=1-f(x)$ for all $x \in X$ is said to be the complement of $f$ in X. In 1999, Smarandache [19] introduced the notion of neutrosophic sets as the following definition. A neutrosophic set (briefly, NS) in a nonempty set $\mathrm{X}$ is a structure of the form:

$$
\Lambda=\left\{\left(x, \lambda_{\mathrm{T}}(x), \lambda_{\mathrm{I}}(x), \lambda_{\mathrm{F}}(x)\right) \mid x \in X\right\},
$$

where $\lambda_{T}: X \rightarrow[0,1]$ is a truth membership function, $\lambda_{I}: X \rightarrow[0,1]$ is an indeterminate membership function, and $\lambda_{\mathrm{F}}: \mathrm{X} \rightarrow[0,1]$ is a false membership function. For our convenience, we will denote a NS as $\Lambda=$ $\left(X, \lambda_{\mathrm{T}}, \lambda_{\mathrm{I}}, \lambda_{\mathrm{F}}\right)=\left(\mathrm{X}, \lambda_{\mathrm{T}, \mathrm{I}, \mathrm{F}}\right)=\left\{\left(\mathrm{x}, \lambda_{\mathrm{T}}(\mathrm{x}), \lambda_{\mathrm{I}}(\mathrm{x}), \lambda_{\mathrm{F}}(\mathrm{x})\right) \mid \mathrm{x} \in \mathrm{X}\right\}$

Definition 2.1 ([19]). Let $\Lambda$ be a NS in a nonempty set $X$. The NS $\bar{\Lambda}=\left(X, \bar{\lambda}_{T, I, F}\right)$ in $X$ defined by

$$
(\forall x \in X)\left(\begin{array}{l}
\bar{\lambda}_{\mathrm{T}}(x)=1-\lambda_{\mathrm{T}}(\mathrm{x}) \\
\bar{\lambda}_{\mathrm{I}}(\mathrm{x})=1-\lambda_{\mathrm{I}}(\mathrm{x}) \\
\bar{\lambda}_{\mathrm{F}}(\mathrm{x})=1-\lambda_{\mathrm{F}}(\mathrm{x})
\end{array}\right)
$$

is called the complement of $\Lambda$ in $X$.

Remark 2.2 ([23]). For all NS $\Lambda$ in a nonempty set $X$, we have $\Lambda=\overline{\bar{\Lambda}}$.

Lemma 2.3 ([27]). Let $\mathrm{a}, \mathrm{b}, \mathrm{c} \in \mathbb{R}$. Then the following statements hold:

(1) $a-\min \{b, c\}=\max \{a-b, a-c\}$, and

(2) $a-\max \{b, c\}=\min \{a-b, a-c\}$.

Lemma 2.4 ([23]). Let $\mathrm{f}$ be a fuzzy set in a nonempty set $\mathrm{X}$. Then the following statements hold:

(1) $(\forall x, y, z \in X)(\bar{f}(x) \geqslant \min \{\bar{f}(y), \bar{f}(z)\} \Leftrightarrow f(x) \leqslant \max \{f(y), f(z)\}) ;$

(2) $(\forall x, y, z \in X)(\bar{f}(x) \leqslant \min \{\bar{f}(y), \bar{f}(z)\} \Leftrightarrow f(x) \geqslant \max \{f(y), f(z)\}) ;$

(3) $(\forall x, y, z \in X)(\bar{f}(x) \geqslant \max \{\bar{f}(y), \bar{f}(z)\} \Leftrightarrow f(x) \leqslant \min \{f(y), f(z)\}) ;$ and

(4) $(\forall x, y, z \in X)(\bar{f}(x) \leqslant \max \{\bar{f}(y), \bar{f}(z)\} \Leftrightarrow f(x) \geqslant \min \{f(y), f(z)\})$.

In what follows, let $X$ denote a UP-algebra $(X, \cdot, 0)$ unless otherwise specified.

Definition 2.5 ([23]). A NS $\wedge$ in $X$ is called a neutrosophic UP-subalgebra of $X$ if it satisfies the following conditions:

$$
\begin{aligned}
& (\forall x, y \in X)\left(\lambda_{T}(x \cdot y) \geqslant \min \left\{\lambda_{T}(x), \lambda_{T}(y)\right\}\right), \\
& (\forall x, y \in X)\left(\lambda_{I}(x \cdot y) \leqslant \max \left\{\lambda_{I}(x), \lambda_{I}(y)\right\}\right), \\
& (\forall x, y \in X)\left(\lambda_{F}(x \cdot y) \geqslant \min \left\{\lambda_{F}(x), \lambda_{F}(y)\right\}\right) .
\end{aligned}
$$


Definition 2.6 ([23]). A NS $\Lambda$ in $X$ is called a neutrosophic near UP-filter of $X$ if it satisfies the following conditions:

$$
\begin{gathered}
(\forall x \in X)\left(\lambda_{\mathrm{T}}(0) \geqslant \lambda_{\mathrm{T}}(x)\right), \\
(\forall x \in X)\left(\lambda_{\mathrm{I}}(0) \leqslant \lambda_{\mathrm{I}}(x)\right), \\
(\forall x \in X)\left(\lambda_{\mathrm{F}}(0) \geqslant \lambda_{\mathrm{F}}(x)\right), \\
(\forall x, y \in X)\left(\lambda_{\mathrm{T}}(x \cdot y) \geqslant \lambda_{\mathrm{T}}(y)\right), \\
(\forall x, y \in X)\left(\lambda_{\mathrm{I}}(x \cdot y) \leqslant \lambda_{\mathrm{I}}(y)\right), \\
(\forall x, y \in X)\left(\lambda_{\mathrm{F}}(x \cdot y) \geqslant \lambda_{\mathrm{F}}(y)\right) .
\end{gathered}
$$

Definition 2.7 ([23]). A NS $\wedge$ in $X$ is called a neutrosophic UP-filter of $X$ if it satisfies the following conditions: (2.6), (2.7), (2.8), and

$$
\begin{aligned}
& (\forall x, y \in X)\left(\lambda_{\mathrm{T}}(y) \geqslant \min \left\{\lambda_{\mathrm{T}}(x \cdot y), \lambda_{\mathrm{T}}(x)\right\}\right), \\
& (\forall x, y \in X)\left(\lambda_{\mathrm{I}}(y) \leqslant \max \left\{\lambda_{\mathrm{I}}(x \cdot y), \lambda_{\mathrm{I}}(x)\right\}\right), \\
& (\forall x, y \in X)\left(\lambda_{\mathrm{F}}(y) \geqslant \min \left\{\lambda_{\mathrm{F}}(x \cdot y), \lambda_{\mathrm{F}}(x)\right\}\right) .
\end{aligned}
$$

Definition 2.8 ([23]). A NS $\Lambda$ in $X$ is called a neutrosophic UP-ideal of $X$ if it satisfies the following conditions: (2.6), (2.7), (2.8), and

$$
\begin{aligned}
& (\forall x, y, z \in X)\left(\lambda_{\mathrm{T}}(x \cdot z) \geqslant \min \left\{\lambda_{\mathrm{T}}(x \cdot(y \cdot z)), \lambda_{\mathrm{T}}(y)\right\}\right), \\
& (\forall x, y, z \in X)\left(\lambda_{\mathrm{I}}(x \cdot z) \leqslant \max \left\{\lambda_{\mathrm{I}}(x \cdot(y \cdot z)), \lambda_{\mathrm{I}}(y)\right\}\right), \\
& (\forall x, y, z \in X)\left(\lambda_{\mathrm{F}}(x \cdot z) \geqslant \min \left\{\lambda_{\mathrm{F}}(x \cdot(y \cdot z)), \lambda_{\mathrm{F}}(y)\right\}\right) .
\end{aligned}
$$

Definition 2.9 ([23]). A NS $\Lambda$ in $X$ is called a neutrosophic strong UP-ideal (renamed from a neutrosophic strongly UP-ideal) of $X$ if it satisfies the following conditions: (2.6), (2.7), (2.8), and

$$
\begin{aligned}
& (\forall x, y, z \in X)\left(\lambda_{\mathrm{T}}(x) \geqslant \min \left\{\lambda_{\mathrm{T}}((z \cdot y) \cdot(z \cdot x)), \lambda_{\mathrm{T}}(y)\right\}\right), \\
& (\forall x, y, z \in X)\left(\lambda_{\mathrm{I}}(x) \leqslant \max \left\{\lambda_{\mathrm{I}}((z \cdot y) \cdot(z \cdot x)), \lambda_{\mathrm{I}}(y)\right\}\right), \\
& (\forall x, y, z \in X)\left(\lambda_{\mathrm{F}}(x) \geqslant \min \left\{\lambda_{\mathrm{F}}((z \cdot y) \cdot(z \cdot x)), \lambda_{\mathrm{F}}(y)\right\}\right) .
\end{aligned}
$$

Definition 2.10 ([23]). A NS $\Lambda$ in $X$ is said to be constant if $\Lambda$ is a constant function from $X$ to $[0,1]^{3}$. That is, $\lambda_{T}, \lambda_{I}$, and $\lambda_{F}$ are constant functions from $X$ to $[0,1]$.

Theorem 2.11 ([23]). A NS $\Lambda$ in $\mathrm{X}$ is constant if and only if it is a neutrosophic strong UP-ideal of X.

Songsaeng and Iampan [23] proved that the notion of neutrosophic UP-subalgebras is a generalization of neutrosophic near UP-filters, neutrosophic near UP-filters is a generalization of neutrosophic UP-filters, neutrosophic UP-filters is a generalization of neutrosophic UP-ideals, and neutrosophic UP-ideals is a generalization of neutrosophic strong UP-ideals.

Now, we introduce the notions of special neutrosophic UP-subalgebras, special neutrosophic near UPfilters, special neutrosophic UP-filters, special neutrosophic UP-ideals, and special neutrosophic strong UP-ideals of UP-algebras, provide the necessary examples, investigate their properties, and prove their generalizations.

Definition 2.12. A NS $\Lambda$ in $X$ is called an special neutrosophic UP-subalgebra of $X$ if it satisfies the following conditions:

$$
\begin{aligned}
& (\forall x, y \in X)\left(\lambda_{T}(x \cdot y) \leqslant \max \left\{\lambda_{T}(x), \lambda_{T}(y)\right\}\right) \\
& (\forall x, y \in X)\left(\lambda_{I}(x \cdot y) \geqslant \min \left\{\lambda_{I}(x), \lambda_{I}(y)\right\}\right), \\
& (\forall x, y \in X)\left(\lambda_{F}(x \cdot y) \leqslant \max \left\{\lambda_{F}(x), \lambda_{F}(y)\right\}\right) .
\end{aligned}
$$


Example 2.13. Let $X=\{0,1,2,3,4\}$ be a UP-algebra with a fixed element 0 and a binary operation $\cdot$ defined by the following Cayley table:

\begin{tabular}{c|ccccc}
$\cdot$ & 0 & 1 & 2 & 3 & 4 \\
\hline 0 & 0 & 1 & 2 & 3 & 4 \\
1 & 0 & 0 & 1 & 0 & 4 \\
2 & 0 & 0 & 0 & 0 & 4 \\
3 & 0 & 1 & 1 & 0 & 4 \\
4 & 0 & 3 & 3 & 3 & 0
\end{tabular}

We define a NS $\wedge$ in $X$ as follows:

$$
\lambda_{\mathrm{T}}=\left(\begin{array}{ccccc}
0 & 1 & 2 & 3 & 4 \\
0.2 & 0.3 & 0.5 & 0.7 & 0.8
\end{array}\right), \lambda_{\mathrm{I}}=\left(\begin{array}{ccccc}
0 & 1 & 2 & 3 & 4 \\
1 & 0.7 & 0.6 & 0.5 & 0.2
\end{array}\right), \lambda_{\mathrm{F}}=\left(\begin{array}{ccccc}
0 & 1 & 2 & 3 & 4 \\
0.1 & 0.4 & 0.6 & 0.7 & 0.9
\end{array}\right) .
$$

Hence, $\Lambda$ is a special neutrosophic UP-subalgebra of $X$.

Definition 2.14. A NS $\Lambda$ in $X$ is called an special neutrosophic near UP-filter of $X$ if it satisfies the following conditions:

$$
\begin{gathered}
(\forall x \in X)\left(\lambda_{\mathrm{T}}(0) \leqslant \lambda_{\mathrm{T}}(x)\right), \\
(\forall x \in X)\left(\lambda_{\mathrm{I}}(0) \geqslant \lambda_{\mathrm{I}}(x)\right), \\
(\forall x \in X)\left(\lambda_{\mathrm{F}}(0) \leqslant \lambda_{\mathrm{F}}(x)\right), \\
(\forall x, y \in X)\left(\lambda_{\mathrm{T}}(x \cdot y) \leqslant \lambda_{\mathrm{T}}(y)\right), \\
(\forall x, y \in X)\left(\lambda_{\mathrm{I}}(x \cdot y) \geqslant \lambda_{\mathrm{I}}(y)\right), \\
(\forall x, y \in X)\left(\lambda_{\mathrm{F}}(x \cdot y) \leqslant \lambda_{\mathrm{F}}(y)\right) .
\end{gathered}
$$

Example 2.15. Let $X=\{0,1,2,3,4\}$ be a UP-algebra with a fixed element 0 and a binary operation $\cdot$ defined by the following Cayley table:

\begin{tabular}{c|ccccc}
$\cdot$ & 0 & 1 & 2 & 3 & 4 \\
\hline 0 & 0 & 1 & 2 & 3 & 4 \\
1 & 0 & 0 & 2 & 2 & 4 \\
2 & 0 & 1 & 0 & 1 & 4 \\
3 & 0 & 0 & 0 & 0 & 4 \\
4 & 0 & 1 & 2 & 3 & 0
\end{tabular}

We define a NS $\wedge$ in $X$ as follows:

$$
\lambda_{\mathrm{T}}=\left(\begin{array}{ccccc}
0 & 1 & 2 & 3 & 4 \\
0 & 0.3 & 0.5 & 0.6 & 0.2
\end{array}\right), \lambda_{\mathrm{I}}=\left(\begin{array}{ccccc}
0 & 1 & 2 & 3 & 4 \\
0.9 & 0.8 & 0.7 & 0.3 & 0.4
\end{array}\right), \lambda_{\mathrm{F}}=\left(\begin{array}{ccccc}
0 & 1 & 2 & 3 & 4 \\
0.1 & 0.2 & 0.6 & 0.7 & 0.5
\end{array}\right) .
$$

Hence, $\Lambda$ is a special neutrosophic near UP-filter of $X$.

Definition 2.16. A NS $\Lambda$ in $X$ is called an special neutrosophic UP-filter of $X$ if it satisfies the following conditions: (2.24), (2.25), (2.26), and

$$
\begin{aligned}
& (\forall x, y \in X)\left(\lambda_{T}(y) \leqslant \max \left\{\lambda_{\mathrm{T}}(x \cdot y), \lambda_{\mathrm{T}}(x)\right\}\right), \\
& (\forall x, y \in X)\left(\lambda_{\mathrm{I}}(y) \geqslant \min \left\{\lambda_{\mathrm{I}}(x \cdot y), \lambda_{\mathrm{I}}(x)\right\}\right), \\
& (\forall x, y \in X)\left(\lambda_{\mathrm{F}}(y) \leqslant \max \left\{\lambda_{\mathrm{F}}(x \cdot y), \lambda_{\mathrm{F}}(x)\right\}\right) .
\end{aligned}
$$

Example 2.17. Let $X=\{0,1,2,3,4\}$ be a UP-algebra with a fixed element 0 and a binary operation $\cdot$ defined by the following Cayley table:

\begin{tabular}{c|ccccc}
$\cdot$ & 0 & 1 & 2 & 3 & 4 \\
\hline 0 & 0 & 1 & 2 & 3 & 4 \\
1 & 0 & 0 & 2 & 2 & 4 \\
2 & 0 & 1 & 0 & 1 & 4 \\
3 & 0 & 0 & 0 & 0 & 4 \\
4 & 0 & 1 & 1 & 1 & 0
\end{tabular}


We define a NS $\Lambda$ in $X$ as follows:

$$
\lambda_{\mathrm{T}}=\left(\begin{array}{ccccc}
0 & 1 & 2 & 3 & 4 \\
0.1 & 0.5 & 0.4 & 0.5 & 0.8
\end{array}\right), \lambda_{\mathrm{I}}=\left(\begin{array}{ccccc}
0 & 1 & 2 & 3 & 4 \\
0.8 & 0.3 & 0.5 & 0.3 & 0.4
\end{array}\right), \lambda_{\mathrm{F}}=\left(\begin{array}{ccccc}
0 & 1 & 2 & 3 & 4 \\
0.2 & 0.6 & 0.4 & 0.6 & 0.3
\end{array}\right) .
$$

Hence, $\Lambda$ is a special neutrosophic UP-filter of $X$.

Definition 2.18. A NS $\Lambda$ in $X$ is called an special neutrosophic UP-ideal of $X$ if it satisfies the following conditions: (2.24), (2.25), (2.26), and

$$
\begin{aligned}
& (\forall x, y, z \in X)\left(\lambda_{\mathrm{T}}(x \cdot z) \leqslant \max \left\{\lambda_{\mathrm{T}}(x \cdot(y \cdot z)), \lambda_{\mathrm{T}}(y)\right\}\right), \\
& (\forall x, y, z \in X)\left(\lambda_{\mathrm{I}}(x \cdot z) \geqslant \min \left\{\lambda_{\mathrm{I}}(x \cdot(y \cdot z)), \lambda_{\mathrm{I}}(y)\right\}\right), \\
& (\forall x, y, z \in X)\left(\lambda_{\mathrm{F}}(x \cdot z) \leqslant \max \left\{\lambda_{\mathrm{F}}(x \cdot(y \cdot z)), \lambda_{\mathrm{F}}(y)\right\}\right) .
\end{aligned}
$$

Example 2.19. Let $X=\{0,1,2,3,4\}$ be a UP-algebra with a fixed element 0 and a binary operation · defined by the following Cayley table:

\begin{tabular}{c|ccccc}
$\cdot$ & 0 & 1 & 2 & 3 & 4 \\
\hline 0 & 0 & 1 & 2 & 3 & 4 \\
1 & 0 & 0 & 2 & 3 & 4 \\
2 & 0 & 0 & 0 & 0 & 4 \\
3 & 0 & 0 & 2 & 0 & 4 \\
4 & 0 & 0 & 0 & 0 & 0
\end{tabular}

We define a NS $\wedge$ in $X$ as follows:

$$
\lambda_{\mathrm{T}}=\left(\begin{array}{ccccc}
0 & 1 & 2 & 3 & 4 \\
0 & 0.3 & 0.5 & 0.4 & 0.6
\end{array}\right), \lambda_{\mathrm{I}}=\left(\begin{array}{ccccc}
0 & 1 & 2 & 3 & 4 \\
1 & 0.7 & 0.4 & 0.7 & 0.3
\end{array}\right), \lambda_{\mathrm{F}}=\left(\begin{array}{ccccc}
0 & 1 & 2 & 3 & 4 \\
0.1 & 0.2 & 0.7 & 0.3 & 0.9
\end{array}\right) .
$$

Hence, $\Lambda$ is a special neutrosophic UP-ideal of $X$.

Definition 2.20. A NS $\Lambda$ in $X$ is called an special neutrosophic strong UP-ideal of $X$ if it satisfies the following conditions: (2.24), (2.25), (2.26), and

$$
\begin{aligned}
& (\forall x, y, z \in X)\left(\lambda_{\mathrm{T}}(x) \leqslant \max \left\{\lambda_{\mathrm{T}}((z \cdot y) \cdot(z \cdot x)), \lambda_{\mathrm{T}}(y)\right\}\right), \\
& (\forall x, y, z \in X)\left(\lambda_{\mathrm{I}}(x) \geqslant \min \left\{\lambda_{\mathrm{I}}((z \cdot y) \cdot(z \cdot x)), \lambda_{\mathrm{I}}(y)\right\}\right), \\
& (\forall x, y, z \in X)\left(\lambda_{\mathrm{F}}(x) \leqslant \max \left\{\lambda_{\mathrm{F}}((z \cdot y) \cdot(z \cdot x)), \lambda_{\mathrm{F}}(y)\right\}\right) .
\end{aligned}
$$

Example 2.21. Let $X=\{0,1,2,3,4\}$ be a UP-algebra with a fixed element 0 and a binary operation $\cdot$ defined by the following Cayley table:

\begin{tabular}{c|ccccc}
$\cdot$ & 0 & 1 & 2 & 3 & 4 \\
\hline 0 & 0 & 1 & 2 & 3 & 4 \\
1 & 0 & 0 & 2 & 3 & 0 \\
2 & 0 & 1 & 0 & 3 & 4 \\
3 & 0 & 1 & 0 & 0 & 4 \\
4 & 0 & 1 & 0 & 3 & 0
\end{tabular}

We define a NS $\Lambda$ in $X$ as follows:

$$
(\forall x \in X)\left(\begin{array}{l}
\lambda_{\mathrm{T}}(x)=0.5 \\
\lambda_{\mathrm{I}}(x)=0.4 \\
\lambda_{\mathrm{F}}(x)=0.7
\end{array}\right)
$$

Hence, $\Lambda$ is a special neutrosophic strong UP-ideal X. 
Theorem 2.22. Every special neutrosophic UP-subalgebra of $X$ satisfies the conditions (2.24), (2.25), and (2.26).

Proof. Assume that $\Lambda$ is a special neutrosophic UP-subalgebra of $X$. Then for all $x \in X$,

$$
\begin{aligned}
& \lambda_{\mathrm{T}}(0)=\lambda_{\mathrm{T}}(\mathrm{x} \cdot \mathrm{x}) \leqslant \max \left\{\lambda_{\mathrm{T}}(\mathrm{x}), \lambda_{\mathrm{T}}(\mathrm{x})\right\}=\lambda_{\mathrm{T}}(\mathrm{x}) \text { by (1.1) and (2.21) } \\
& \lambda_{\mathrm{I}}(0)=\lambda_{\mathrm{I}}(x \cdot x) \geqslant \min \left\{\lambda_{\mathrm{I}}(x), \lambda_{\mathrm{I}}(x)\right\}=\lambda_{\mathrm{I}}(x) \text { by (1.1) and (2.22) } \\
& \lambda_{\mathrm{F}}(0)=\lambda_{\mathrm{F}}(\mathrm{x} \cdot \mathrm{x}) \leqslant \max \left\{\lambda_{\mathrm{F}}(\mathrm{x}), \lambda_{\mathrm{F}}(\mathrm{x})\right\}=\lambda_{\mathrm{F}}(\mathrm{x}) \text { by (1.1) and (2.23). }
\end{aligned}
$$

Hence, $\wedge$ satisfies the conditions (2.24), (2.25), and (2.26).

By Lemma 2.4 (1) and (4), we have the following five theorems.

Theorem 2.23. A NS $\Lambda$ in $\mathrm{X}$ is a neutrosophic UP-subalgebra of $\mathrm{X}$ if and only if $\bar{\Lambda}$ is a special neutrosophic UP-subalgebra of $\mathrm{X}$.

Theorem 2.24. A NS $\Lambda$ in $X$ is a neutrosophic near UP-filter of $X$ if and only if $\bar{\Lambda}$ is a special neutrosophic near UP-filter of $X$.

Theorem 2.25. A NS $\Lambda$ in $X$ is a neutrosophic UP-filter of $X$ if and only if $\bar{\Lambda}$ is a special neutrosophic UP-filter of $X$.

Theorem 2.26. A NS $\wedge$ in $\mathrm{X}$ is a neutrosophic UP-ideal of $\mathrm{X}$ if and only if $\bar{\Lambda}$ is a special neutrosophic UP-ideal of $X$.

Theorem 2.27. A NS $\Lambda$ in $\mathrm{X}$ is a neutrosophic strong UP-ideal of $\mathrm{X}$ if and only if $\bar{\Lambda}$ is a special neutrosophic strong UP-ideal of $\mathrm{X}$.

Theorem 2.28. A NS $\Lambda$ in $\mathrm{X}$ is constant if and only if it is a special neutrosophic strong UP-ideal of X.

Proof. It is straightforward by Remark 2.2 and Theorems 2.11 and 2.27.

By Remark 2.2 and Theorems 2.23, 2.24, 2.25, 2.26, and 2.27, we have that the notion of special neutrosophic UP-subalgebras is a generalization of special neutrosophic near UP-filters, special neutrosophic near UP-filters is a generalization of special neutrosophic UP-filters, special neutrosophic UP-filters is a generalization of special neutrosophic UP-ideals, and special neutrosophic UP-ideals is a generalization of special neutrosophic strong UP-ideals. Moreover, by Theorem 2.28, we obtain that special neutrosophic strong UP-ideals and constant neutrosophic set coincide.

Theorem 2.29. If $\Lambda$ is a special neutrosophic UP-subalgebra of $X$ satisfying the following condition:

$$
(\forall x, y \in X)\left(x \cdot y \neq 0 \Rightarrow\left\{\begin{array}{l}
\lambda_{\mathrm{T}}(x) \leqslant \lambda_{\mathrm{T}}(y) \\
\lambda_{\mathrm{I}}(x) \geqslant \lambda_{\mathrm{I}}(y) \\
\lambda_{\mathrm{F}}(x) \leqslant \lambda_{\mathrm{F}}(y)
\end{array}\right),\right.
$$

then $\Lambda$ is a special neutrosophic near UP-filter of $X$.

Proof. Assume that $\Lambda$ is a special neutrosophic UP-subalgebra of $X$ satisfying the condition (2.39). By Theorem 2.22, we have $\Lambda$ satisfies the conditions (2.24), (2.25), and (2.26). Next, let $x, y \in X$.

Case 1: $x \cdot y=0$. Then

$$
\begin{aligned}
& \lambda_{T}(x \cdot y)=\lambda_{T}(0) \leqslant \lambda_{T}(y) \text { by }(2.24) \\
& \lambda_{I}(x \cdot y)=\lambda_{I}(0) \geqslant \lambda_{I}(y) \text { by }(2.25) \\
& \lambda_{F}(x \cdot y)=\lambda_{F}(0) \leqslant \lambda_{F}(y) \text { by }(2.26) .
\end{aligned}
$$


Case 2: $x \cdot y \neq 0$. Then

$$
\begin{aligned}
& \lambda_{T}(x \cdot y) \leqslant \max \left\{\lambda_{T}(x), \lambda_{T}(y)\right\}=\lambda_{T}(y), \quad(2.21) \text { and (2.39) for } \lambda_{T}, \\
& \lambda_{I}(x \cdot y) \geqslant \min \left\{\lambda_{I}(x), \lambda_{I}(y)\right\}=\lambda_{I}(y), \quad(2.22) \text { and (2.39) for } \lambda_{I}, \\
& \lambda_{F}(x \cdot y) \leqslant \max \left\{\lambda_{F}(x), \lambda_{F}(y)\right\}=\lambda_{F}(y) . \quad(2.23) \text { and (2.39) for } \lambda_{F} .
\end{aligned}
$$

Hence, $\Lambda$ is a special neutrosophic near UP-filter of $X$.

Theorem 2.30. If $\Lambda$ is a special neutrosophic near UP-filter of $X$ satisfying the following condition:

$$
\lambda_{\mathrm{T}}=\lambda_{\mathrm{I}}=\lambda_{\mathrm{F}}
$$

then $\Lambda$ is a special neutrosophic UP-filter of $X$.

Proof. Assume that $\Lambda$ is a special neutrosophic near UP-filter of $X$ satisfying the condition (2.40). Then $\Lambda$ satisfies the conditions (2.24), (2.25), and (2.26). Next, let $x, y, z \in X$. Then

$$
\begin{aligned}
\max \left\{\lambda_{\mathrm{T}}(x \cdot y), \lambda_{\mathrm{T}}(x)\right\} & =\max \left\{\lambda_{\mathrm{I}}(x \cdot y), \lambda_{\mathrm{T}}(x)\right\} \quad \text { by }(2.40) \\
& \geqslant \max \left\{\lambda_{\mathrm{I}}(y), \lambda_{\mathrm{T}}(x)\right\} \quad \text { by }(2.28) \\
& =\max \left\{\lambda_{\mathrm{T}}(y), \lambda_{\mathrm{T}}(x)\right\} \quad \text { by }(2.40) \\
& \geqslant \lambda_{\mathrm{T}}(y), \\
\min \left\{\lambda_{\mathrm{I}}(x \cdot y), \lambda_{\mathrm{I}}(x)\right\} & =\min \left\{\lambda_{\mathrm{T}}(x \cdot y), \lambda_{\mathrm{I}}(x)\right\} \quad \text { by }(2.40) \\
& \leqslant \min \left\{\lambda_{\mathrm{T}}(y), \lambda_{\mathrm{I}}(x)\right\} \quad \text { by }(2.27) \\
& =\min \left\{\lambda_{\mathrm{I}}(y), \lambda_{\mathrm{I}}(x)\right\} \quad \text { by }(2.40) \\
& \leqslant \lambda_{\mathrm{I}}(y), \\
\max \left\{\lambda_{\mathrm{F}}(x \cdot y), \lambda_{\mathrm{F}}(x)\right\} & =\max \left\{\lambda_{\mathrm{I}}(x \cdot y), \lambda_{\mathrm{F}}(x)\right\} \quad \text { by }(2.40) \\
& \geqslant \max \left\{\lambda_{\mathrm{I}}(y), \lambda_{\mathrm{F}}(x)\right\} \quad \text { by }(2.28) \\
& =\max \left\{\lambda_{\mathrm{F}}(y), \lambda_{\mathrm{F}}(x)\right\} \quad \text { by }(2.40) \\
& \geqslant \lambda_{\mathrm{F}}(y) .
\end{aligned}
$$

Hence, $\Lambda$ is a special neutrosophic UP-filter of $X$.

Theorem 2.31. If $\Lambda$ is a special neutrosophic UP-filter of $X$ satisfying the following condition:

$$
(\forall x, y, z \in X)\left(\begin{array}{l}
\lambda_{\mathrm{T}}(y \cdot(x \cdot z))=\lambda_{\mathrm{T}}(x \cdot(y \cdot z)) \\
\lambda_{\mathrm{I}}(y \cdot(x \cdot z))=\lambda_{\mathrm{I}}(x \cdot(y \cdot z)) \\
\lambda_{\mathrm{F}}(y \cdot(x \cdot z))=\lambda_{\mathrm{F}}(x \cdot(y \cdot z))
\end{array}\right)
$$

then $\Lambda$ is a special neutrosophic UP-ideal of $X$.

Proof. Assume that $\Lambda$ is a special neutrosophic UP-filter of $X$ satisfying the condition (2.41). Then $\Lambda$ satisfies the conditions (2.24), (2.25), and (2.26). Next, let $x, y, z \in X$. Then

$$
\begin{aligned}
\lambda_{\mathrm{T}}(x \cdot z) & \leqslant \max \left\{\lambda_{\mathrm{T}}(y \cdot(x \cdot z)), \lambda_{\mathrm{T}}(y)\right\} \quad \text { by }(2.30) \\
& =\max \left\{\lambda_{\mathrm{T}}(x \cdot(y \cdot z)), \lambda_{\mathrm{T}}(y)\right\} \quad \text { by }(2.41) \text { for } \lambda_{\mathrm{T}} \\
\lambda_{\mathrm{I}}(x \cdot z) & \geqslant \min \left\{\lambda_{\mathrm{I}}(y \cdot(x \cdot z)), \lambda_{\mathrm{I}}(y)\right\} \quad \text { by }(2.31) \\
& =\min \left\{\lambda_{\mathrm{I}}(x \cdot(y \cdot z)), \lambda_{\mathrm{I}}(y)\right\} \quad \text { by }(2.41) \text { for } \lambda_{\mathrm{I}} \\
\lambda_{\mathrm{F}}(x \cdot z) & \leqslant \max \left\{\lambda_{\mathrm{F}}(y \cdot(x \cdot z)), \lambda_{\mathrm{F}}(y)\right\} \quad \text { by }(2.32) \\
& =\max \left\{\lambda_{\mathrm{F}}(x \cdot(y \cdot z)), \lambda_{\mathrm{F}}(y)\right\} \quad \text { by }(2.41) \text { for } \lambda_{\mathrm{F}} .
\end{aligned}
$$

Hence, $\Lambda$ is a special neutrosophic UP-ideal of $X$. 
Theorem 2.32. If $\Lambda$ is a NS in $\mathrm{X}$ satisfying the following condition:

$$
(\forall x, y, z \in X)\left(z \leqslant x \cdot y \Rightarrow\left\{\begin{array}{l}
\lambda_{\mathrm{T}}(z) \leqslant \max \left\{\lambda_{\mathrm{T}}(x), \lambda_{\mathrm{T}}(y)\right\} \\
\lambda_{\mathrm{I}}(z) \geqslant \min \left\{\lambda_{\mathrm{I}}(x), \lambda_{\mathrm{I}}(y)\right\} \\
\lambda_{\mathrm{F}}(z) \leqslant \max \left\{\lambda_{\mathrm{F}}(x), \lambda_{\mathrm{F}}(y)\right\}
\end{array}\right)\right.
$$

then $\Lambda$ is a special neutrosophic UP-subalgebra of $\mathrm{X}$.

Proof. Assume that $\Lambda$ is a NS in $X$ satisfying the condition (2.42). Let $x, y \in X$. By (1.1), we have $(x \cdot y) \cdot(x \cdot y)=0$, that is, $x \cdot y \geqslant x \cdot y$. It follows from $(2.42)$ that

$$
\lambda_{T}(x \cdot y) \leqslant \max \left\{\lambda_{T}(x), \lambda_{T}(y)\right\}, \quad \lambda_{I}(x \cdot y) \geqslant \min \left\{\lambda_{I}(x), \lambda_{I}(y)\right\}, \quad \lambda_{F}(x \cdot y) \leqslant \max \left\{\lambda_{F}(x), \lambda_{F}(y)\right\} .
$$

Hence, $\Lambda$ is a special neutrosophic UP-subalgebra of $X$.

Theorem 2.33. If $\Lambda$ is a NS in X satisfying the following condition:

$$
(\forall x, y, z \in X)\left(z \leqslant x \cdot y \Rightarrow\left\{\begin{array}{l}
\lambda_{\mathrm{T}}(z) \leqslant \lambda_{\mathrm{T}}(y) \\
\lambda_{\mathrm{I}}(z) \geqslant \lambda_{\mathrm{I}}(y) \\
\lambda_{\mathrm{F}}(z) \leqslant \lambda_{\mathrm{F}}(y)
\end{array}\right)\right.
$$

then $\Lambda$ is a special neutrosophic near UP-filter of $X$.

Proof. Assume that $\Lambda$ is a NS in $X$ satisfying the condition (2.43). Let $x \in X$. By (UP-2) and (1.1), we have $0 \cdot(x \cdot x)=0$, that is, $0 \leqslant x \cdot x$. It follows from $(2.43)$ that $\lambda_{T}(0) \leqslant \lambda_{T}(x), \lambda_{I}(0) \geqslant \lambda_{I}(x)$, and $\lambda_{F}(0) \leqslant \lambda_{F}(x)$. Next, let $x, y \in X$. By $(1.1)$, we have $(x \cdot y) \cdot(x \cdot y)=0$, that is, $x \cdot y \geqslant x \cdot y$. It follows from (2.43) that $\lambda_{T}(x \cdot y) \leqslant \lambda_{T}(y), \lambda_{I}(x \cdot y) \geqslant \lambda_{I}(y)$, and $\lambda_{F}(x \cdot y) \leqslant \lambda_{F}(y)$. Hence, $\Lambda$ is a special neutrosophic near UP-filter of $X$.

Theorem 2.34. If $\Lambda$ is a NS in X satisfying the following condition:

$$
(\forall x, y, z \in X)\left(z \leqslant x \cdot y \Rightarrow\left\{\begin{array}{l}
\lambda_{\mathrm{T}}(y) \leqslant \max \left\{\lambda_{\mathrm{T}}(z), \lambda_{\mathrm{T}}(x)\right\} \\
\lambda_{\mathrm{I}}(y) \geqslant \min \left\{\lambda_{\mathrm{I}}(z), \lambda_{\mathrm{I}}(x)\right\} \\
\lambda_{\mathrm{F}}(y) \leqslant \max \left\{\lambda_{\mathrm{F}}(z), \lambda_{\mathrm{F}}(x)\right\}
\end{array}\right)\right.
$$

then $\Lambda$ is a special neutrosophic UP-filter of $X$.

Proof. Assume that $\Lambda$ is a NS in $X$ satisfying the condition (2.44). Let $x \in X$. By (UP-3), we have $x \cdot(x \cdot 0)=$ 0 , that is, $x \leqslant x \cdot 0$. It follows from $(2.44)$ that

$\lambda_{\mathrm{T}}(0) \leqslant \max \left\{\lambda_{\mathrm{T}}(\mathrm{x}), \lambda_{\mathrm{T}}(\mathrm{x})\right\}=\lambda_{\mathrm{T}}(\mathrm{x}), \lambda_{\mathrm{I}}(0) \geqslant \min \left\{\lambda_{\mathrm{I}}(\mathrm{x}), \lambda_{\mathrm{I}}(\mathrm{x})\right\}=\lambda_{\mathrm{I}}(\mathrm{x}), \lambda_{\mathrm{F}}(0) \leqslant \max \left\{\lambda_{\mathrm{F}}(\mathrm{x}), \lambda_{\mathrm{F}}(\mathrm{x})\right\}=\lambda_{\mathrm{F}}(\mathrm{x})$.

Next, let $x, y \in X$. By (1.1), we have $(x \cdot y) \cdot(x \cdot y)=0$, that is, $x \cdot y \geqslant x \cdot y$. It follows from (2.44) that

$$
\lambda_{T}(y) \leqslant \max \left\{\lambda_{T}(x \cdot y), \lambda_{T}(x)\right\}, \quad \lambda_{I}(y) \geqslant \min \left\{\lambda_{I}(x \cdot y), \lambda_{I}(x)\right\}, \quad \lambda_{F}(y) \leqslant \max \left\{\lambda_{F}(x \cdot y), \lambda_{F}(x)\right\} .
$$

Hence, $\Lambda$ is a special neutrosophic UP-filter of $X$.

Theorem 2.35. If $\Lambda$ is a NS in X satisfying the following condition:

$$
(\forall a, x, y, z \in X)\left(a \leqslant x \cdot(y \cdot z) \Rightarrow\left\{\begin{array}{l}
\lambda_{\mathrm{T}}(x \cdot z) \leqslant \max \left\{\lambda_{\mathrm{T}}(a), \lambda_{\mathrm{T}}(y)\right\} \\
\lambda_{\mathrm{I}}(x \cdot z) \geqslant \min \left\{\lambda_{\mathrm{I}}(a), \lambda_{\mathrm{I}}(y)\right\} \\
\lambda_{\mathrm{F}}(x \cdot z) \leqslant \max \left\{\lambda_{\mathrm{F}}(a), \lambda_{\mathrm{F}}(y)\right\}
\end{array}\right),\right.
$$

then $\Lambda$ is a special neutrosophic UP-ideal of X. 
Proof. Assume that $\Lambda$ is a NS in $X$ satisfying the condition (2.45). Let $x \in X$. By (UP-3), we have $x \cdot(0 \cdot(x$. $0))=0$, that is, $x \leqslant 0 \cdot(x \cdot 0)$. It follows from $(2.45)$ that

$$
\begin{aligned}
& \lambda_{\mathrm{T}}(0)=\lambda_{\mathrm{T}}(0 \cdot 0) \leqslant \max \left\{\lambda_{\mathrm{T}}(x), \lambda_{\mathrm{T}}(x)\right\}=\lambda_{\mathrm{T}}(x), \\
& \lambda_{\mathrm{I}}(0)=\lambda_{\mathrm{I}}(0 \cdot 0) \geqslant \min \left\{\lambda_{\mathrm{I}}(x), \lambda_{\mathrm{I}}(x)\right\}=\lambda_{\mathrm{I}}(x), \\
& \lambda_{\mathrm{F}}(0)=\lambda_{\mathrm{F}}(0 \cdot 0) \leqslant \max \left\{\lambda_{\mathrm{F}}(x), \lambda_{\mathrm{F}}(x)\right\}=\lambda_{\mathrm{F}}(x) .
\end{aligned}
$$

Next, let $x, y, z \in X$. By (1.1), we have $(x \cdot(y \cdot z)) \cdot(x \cdot(y \cdot z))=0$, that is, $x \cdot(y \cdot z) \geqslant x \cdot(y \cdot z)$. It follows from (2.45) that

$$
\begin{aligned}
& \lambda_{T}(x \cdot z) \leqslant \max \left\{\lambda_{T}(x \cdot(y \cdot z)), \lambda_{T}(y)\right\}, \\
& \lambda_{I}(x \cdot z) \geqslant \min \left\{\lambda_{I}(x \cdot(y \cdot z)), \lambda_{I}(y)\right\}, \\
& \lambda_{F}(x \cdot z) \leqslant \max \left\{\lambda_{F}(x \cdot(y \cdot z)), \lambda_{F}(y)\right\} .
\end{aligned}
$$

Hence, $\Lambda$ is a special neutrosophic UP-ideal of $X$.

For any fixed numbers $\alpha^{+}, \alpha^{-}, \beta^{+}, \beta^{-}, \gamma^{+}, \gamma^{-} \in[0,1]$ such that $\alpha^{+}<\alpha^{-}, \beta^{+}<\beta^{-}, \gamma^{+}<\gamma^{-}$and a nonempty subset $G$ of $X$, a NS $\Lambda^{G}\left[\begin{array}{c}\alpha^{+}, \beta^{-}, \gamma^{+} \\ \alpha^{-}, \beta^{+}, \gamma^{-}\end{array}\right]=\left(X, \lambda_{T}^{G}\left[\begin{array}{c}\alpha^{+} \\ \alpha^{-}\end{array}\right], \lambda_{I}^{G}\left[\begin{array}{c}\beta^{-} \\ \beta^{+}\end{array}\right], \lambda_{F}^{G}\left[\begin{array}{c}\gamma^{+} \\ \gamma^{-}\end{array}\right)\right.$in $X$, where $\lambda_{T}^{G}\left[\begin{array}{c}\alpha^{+} \\ \alpha^{-}\end{array}, \lambda_{I}^{G}\left[\begin{array}{c}\beta^{-} \\ \beta^{+}\end{array}\right]\right.$, and $\lambda_{\mathrm{F}}^{\mathrm{G}}\left[\gamma_{\gamma^{+}}^{\gamma^{+}}\right]$are functions on $\mathrm{X}$ which are given as follows:

$$
\lambda_{T}^{G}\left[\alpha^{\alpha^{+}}\right](x)=\left\{\begin{array}{ll}
\alpha^{+}, & \text {if } x \in G, \\
\alpha^{-}, & \text {otherwise, }
\end{array} \quad \lambda_{\mathrm{I}}^{G}\left[_{\beta^{+}}^{\beta^{-}}\right](x)=\left\{\begin{array}{ll}
\beta^{-}, & \text {if } x \in G, \\
\beta^{+}, & \text {otherwise, }
\end{array} \quad \lambda_{F}^{G} \gamma_{\gamma^{-}}^{\gamma^{+}}\right](x)= \begin{cases}\gamma^{+}, & \text {if } x \in G, \\
\gamma^{-}, & \text {otherwise. }\end{cases}\right.
$$

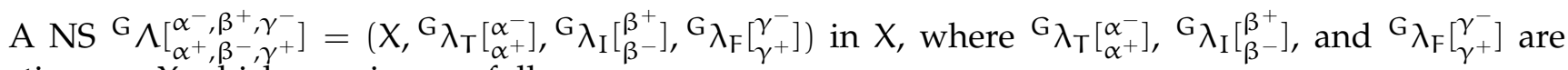
functions on $X$ which are given as follows:

$$
{ }^{G} \lambda_{T}\left[\alpha_{\alpha^{+}}^{\alpha^{-}}\right](x)=\left\{\begin{array}{ll}
\alpha^{-}, & \text {if } x \in G, \\
\alpha^{+}, & \text {otherwise, }
\end{array} G^{G} \lambda_{I}\left[\beta_{\beta^{-}}^{\beta^{+}}\right](x)=\left\{\begin{array}{ll}
\beta^{+}, & \text {if } x \in G, \quad G \\
\beta^{-}, & \text {otherwise, }
\end{array}{ }^{G} \lambda_{F} r_{\gamma^{+}}^{\gamma^{-}}\right](x)= \begin{cases}\gamma^{-}, & \text {if } x \in G, \\
\gamma^{+}, & \text {otherwise. }\end{cases}\right.
$$

Lemma 2.36. Let $\alpha^{+}, \alpha^{-}, \beta^{+}, \beta^{-}, \gamma^{+}, \gamma^{-} \in[0,1]$. Then the following statements hold:

(1) $\overline{\Lambda^{\mathrm{G}}\left[\begin{array}{c}\alpha^{+}, \beta^{-}, \gamma^{+} \\ \alpha^{-}, \beta^{+}, \gamma^{-}\end{array}\right]}={ }^{\mathrm{G}} \wedge\left[\begin{array}{c}1-\alpha^{+}, 1-\beta^{-}, 1-\gamma^{+} \\ 1-\alpha^{-}, 1-\beta^{+}, 1-\gamma^{-}\end{array}\right]$, and

(2) $\overline{\mathrm{G} \Lambda \Lambda_{\alpha^{+}, \beta^{-}, \gamma^{+}}^{\alpha^{-}, \beta^{+}, \gamma^{-}}}=\Lambda^{\mathrm{G}}\left[\begin{array}{c}1-\alpha^{-}, 1-\beta^{+}, 1-\gamma^{-} \\ 1-\alpha^{+}, 1-\beta^{-}, 1-\gamma^{+}\end{array}\right]$

Proof.

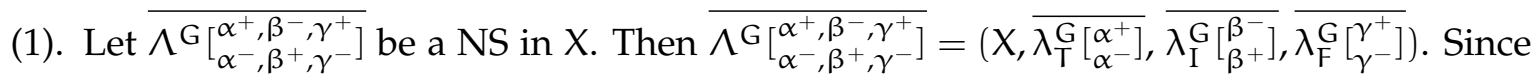

$$
\lambda_{T}^{G}\left[\alpha^{\alpha^{+}}\right](x)=\left\{\begin{array}{ll}
\alpha^{+}, & \text {if } x \in G, \\
\alpha^{-}, & \text {otherwise, }
\end{array} \quad \lambda_{I}^{G}\left[\beta_{\beta^{+}}^{\beta^{-}}\right](x)=\left\{\begin{array}{ll}
\beta^{-}, & \text {if } x \in G, \\
\beta^{+}, & \text {otherwise, }
\end{array} \quad \lambda_{F}^{G} \gamma_{\gamma^{-}}^{\gamma^{+}}\right](x)= \begin{cases}\gamma^{+}, & \text {if } x \in G, \\
\gamma^{-}, & \text {otherwise. }\end{cases}\right.
$$

Thus

$$
\begin{aligned}
& \overline{\left.\lambda_{\mathrm{T}}^{\mathrm{G}}{ }_{\alpha^{-}}^{\alpha^{+}}\right]}(x)=\left\{\begin{array}{ll}
1-\alpha^{+}, & \text {if } x \in \mathrm{G}, \\
1-\alpha^{-}, & \text {otherwise }
\end{array}={ }^{\mathrm{G}} \lambda_{\mathrm{T}}\left[\begin{array}{ll}
1-\alpha^{+} \\
1-\alpha^{-}
\end{array}\right](x),\right. \\
& \overline{\lambda_{\mathrm{I}}^{\mathrm{G}}\left[{ }_{\beta^{+}}^{\beta^{-}}\right]}(x)=\left\{\begin{array}{ll}
1-\beta^{-}, & \text {if } x \in \mathrm{G}, \\
1-\beta^{+}, & \text {otherwise }
\end{array}={ }^{\mathrm{G}} \lambda_{\mathrm{I}}\left[\begin{array}{ll}
1-\beta^{-} \\
1-\beta^{+}
\end{array}\right](x),\right. \\
& \overline{\left.\lambda_{\mathrm{F}}^{\mathrm{G}} \gamma_{\gamma^{+}}^{\gamma^{+}}\right]}(x)=\left\{\begin{array}{ll}
1-\gamma^{+}, & \text {if } x \in \mathrm{G}, \\
1-\gamma^{-}, & \text {otherwise }
\end{array}={ }^{\mathrm{G}} \lambda_{\mathrm{F}}\left[\begin{array}{l}
1-\gamma^{+} \\
1-\gamma^{-}
\end{array}\right](x) .\right.
\end{aligned}
$$

Hence, $\left(X,{ }^{G} \lambda_{T}\left[\begin{array}{l}1-\alpha^{+} \\ 1-\alpha^{-}\end{array},{ }^{G} \lambda_{I}\left[\begin{array}{l}1-\beta^{-} \\ 1-\beta^{+}\end{array}\right],{ }^{G} \lambda_{F}\left[\begin{array}{l}1-\gamma^{+} \\ 1-\gamma^{-}\end{array}\right]\right)={ }^{G} \wedge\left[\begin{array}{c}1-\alpha^{+}, 1-\beta^{-}, 1-\gamma^{+} \\ 1-\alpha^{-}, 1-\beta^{+}, 1-\gamma^{-}\end{array}\right]\right.$. 


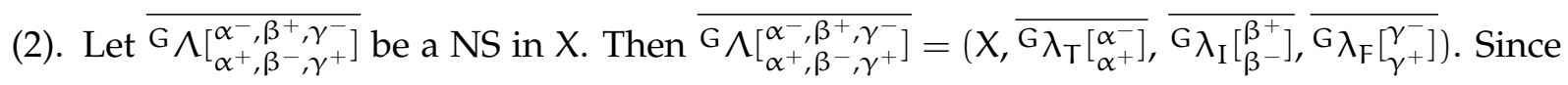

$$
{ }^{G} \lambda_{T}\left[\begin{array}{ll}
\alpha^{-} \\
\alpha^{+}
\end{array}\right](x)=\left\{\begin{array}{ll}
\alpha^{-}, & \text {if } x \in G, \\
\alpha^{+}, & \text {otherwise, }
\end{array}{ }^{G} \lambda_{I_{I}}\left[\beta_{\beta^{+}}^{\beta^{+}}\right](x)=\left\{\begin{array}{ll}
\beta^{+}, & \text {if } x \in G, \quad G \\
\beta^{-}, & \text {otherwise, }
\end{array}{ }^{G} \lambda_{F}\left[\gamma_{\gamma^{+}}^{\gamma^{-}}\right](x)= \begin{cases}\gamma^{-}, & \text {if } x \in G, \\
\gamma^{+}, & \text {otherwise. }\end{cases}\right.\right.
$$

Thus

$$
\begin{aligned}
& \overline{{ }^{\mathrm{G}} \lambda_{\mathrm{T}}\left[\alpha_{\alpha^{+}}^{\alpha^{-}}\right]}(x)=\left\{\begin{array}{ll}
1-\alpha^{-}, & \text {if } x \in \mathrm{G}, \\
1-\alpha^{+}, & \text {otherwise }
\end{array}=\lambda_{\mathrm{T}}^{\mathrm{G}}\left[1-\alpha^{+}\right](x),\right. \\
& \left.\overline{{ }^{G} \lambda_{I}\left[\beta^{-} \beta^{+}\right.}\right](x)=\left\{\begin{array}{ll}
1-\beta^{+}, & \text {if } x \in G, \\
1-\beta^{-}, & \text {otherwise }
\end{array}=\lambda_{I}^{G}\left[\begin{array}{ll}
1-\beta^{+} \\
1-\beta^{-}
\end{array}\right](x),\right. \\
& \overline{{ }^{G} \lambda_{F}\left[_{\gamma^{+}}^{\gamma^{-}}\right]}(x)=\left\{\begin{array}{ll}
1-\gamma^{-}, & \text {if } x \in G, \\
1-\gamma^{+}, & \text {otherwise }
\end{array}=\lambda_{F}^{G}\left[\begin{array}{l}
1-\gamma^{-} \\
1-\gamma^{+}
\end{array}\right](x)\right. \text {. }
\end{aligned}
$$

Hence, $\left(X, \lambda_{T}^{G}\left[\begin{array}{l}1-\alpha^{-} \\ 1-\alpha^{+}\end{array}\right], \lambda_{I}^{G}\left[\begin{array}{l}1-\beta^{+} \\ 1-\beta^{-}\end{array}\right], \lambda_{F}^{G}\left[\begin{array}{l}1-\gamma^{-} \\ 1-\gamma^{+}\end{array}\right]\right)=\Lambda^{G}\left[\begin{array}{c}1-\alpha^{-}, 1-\beta^{+}, 1-\gamma^{-} \\ 1-\alpha^{+}, 1-\beta^{-}, 1-\gamma^{+}\end{array}\right]$.

Lemma 2.37. If the constant 0 of $X$ is in a nonempty subset $G$ of $X$, then a $N S G \Lambda_{\left[\alpha^{+}, \beta^{-}, \gamma^{+}\right.}^{\alpha^{-}, \beta^{+}, \gamma^{-}}$in $X$ satisfies the conditions (2.24), (2.25), and (2.26).

Proof. If $0 \in \mathrm{G}$, then ${ }^{\mathrm{G}} \lambda_{\mathrm{T}}\left[{ }_{\alpha^{+}}^{\alpha^{-}}\right](0)=\alpha^{-},{ }^{\mathrm{G}} \lambda_{\mathrm{I}}\left[{ }_{\beta^{-}}^{\beta^{+}}\right](0)=\beta^{+}$, and ${ }^{\mathrm{G}} \lambda_{\mathrm{F}}\left[_{\gamma^{+}}^{\gamma^{-}}\right](0)=\gamma^{-}$. Thus

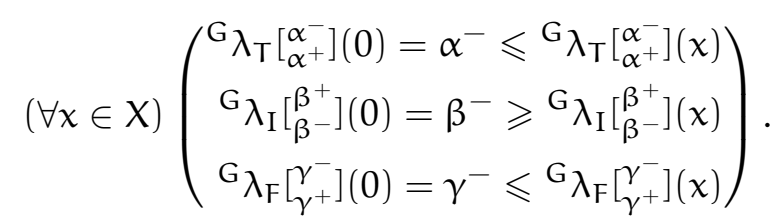

Hence, ${ }^{G} \wedge\left[\begin{array}{c}\alpha^{-}, \beta^{+}, \gamma^{-} \\ \alpha^{+}, \beta^{-}, \gamma^{+}\end{array}\right]$satisfies the conditions (2.24), (2.25), and (2.26).

Lemma 2.38. If a NS $\mathrm{G}^{\mathrm{N}}\left[\begin{array}{c}\alpha^{-}, \beta^{+}, \gamma^{-} \\ \alpha^{+}, \beta^{-}, \gamma^{+}\end{array}\right]$in $\mathrm{X}$ satisfies the condition (2.24) (resp., (2.25), (2.26)), then the constant 0 of $X$ is in a nonempty subset $\mathrm{G}$ of $X^{\prime}$.

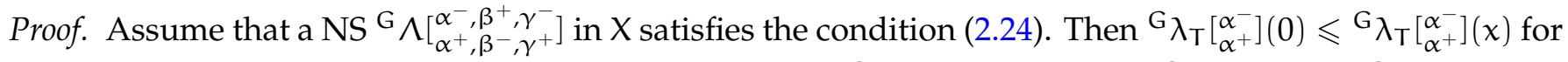
all $x \in X$. Since $G$ is nonempty, there exists $g \in G$. Thus ${ }^{G} \lambda_{T}\left[\alpha_{\alpha^{+}}^{\alpha^{-}}\right](g)=\alpha^{-}$, so ${ }^{G} \lambda_{T}\left[\alpha_{\alpha^{+}}^{\alpha^{-}}\right](0) \leqslant{ }^{G} \lambda_{T}\left[\alpha_{\alpha^{+}}^{\alpha^{-}}\right](g)=$ $\alpha^{-}$, that is, ${ }^{\mathrm{G}} \lambda_{\mathrm{T}}\left[\begin{array}{l}\alpha^{-} \\ \alpha^{+}\end{array}\right](0)=\alpha^{-}$. Hence, $0 \in \mathrm{G}$.

Theorem 2.39. A NS $\mathrm{G} \wedge \Lambda_{\alpha^{+}, \beta^{-}, \gamma^{+}}^{\alpha^{-}, \beta^{+}, \gamma^{-}}$in $\mathrm{X}$ is a special neutrosophic UP-subalgebra of $\mathrm{X}$ if and only if a nonempty subset $\mathrm{G}$ of $\mathrm{X}$ is a UP-subalgebra of $\mathrm{X}$.

Proof. Assume that ${ }^{G} \Lambda_{\left[\alpha^{+}, \beta^{-}, \gamma^{+}\right.}^{\alpha^{-}, \beta^{+}, \gamma^{-}}$is a special neutrosophic UP-subalgebra of $X$. Let $x, y \in G$. Then ${ }^{\mathrm{G}} \lambda_{\mathrm{T}}\left[\alpha^{\alpha^{-}}\right](\mathrm{x})=\alpha^{-}={ }^{\mathrm{G}} \lambda_{\mathrm{T}}\left[{ }_{\alpha^{+}}^{\alpha^{-}}\right](\mathrm{y})$. Thus

$$
{ }^{G} \lambda_{T}\left[{ }_{\alpha^{+}}^{\alpha^{-}}\right](x \cdot y) \leqslant \max \left\{{ }^{G} \lambda_{T}\left[\alpha_{\alpha^{+}}^{\alpha^{-}}\right](x),{ }^{G} \lambda_{T}\left[{ }_{\alpha^{+}}^{\alpha^{-}}\right](y)\right\}=\alpha^{-} \leqslant{ }^{G} \lambda_{T}\left[\alpha_{\alpha^{+}}^{\alpha^{-}}\right](x \cdot y) \text { by }(2.21)
$$

and so ${ }^{G} \lambda_{\mathrm{T}}\left[{ }_{\alpha^{+}}^{\alpha^{-}}\right](x \cdot y)=\alpha^{-}$. Thus $x \cdot y \in G$. Hence, $G$ is a UP-subalgebra of $X$.

Conversely, assume that $G$ is a UP-subalgebra of $X$. Let $x, y \in X$. 
Case 1: $x, y \in G$. Then

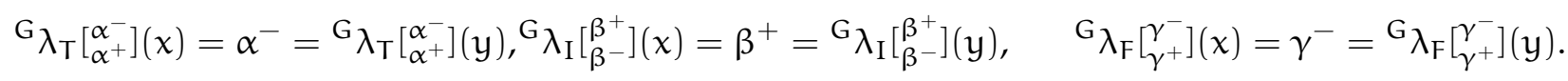

Thus

$$
\begin{gathered}
\max \left\{{ }^{G} \lambda_{\mathrm{T}}\left[\begin{array}{c}
\alpha^{-} \\
\alpha^{+}
\end{array}\right](x),{ }^{G} \lambda_{\mathrm{T}}\left[\begin{array}{l}
\alpha^{-} \\
\alpha^{+}
\end{array}\right](y)\right\}=\alpha^{-}, \\
\min \left\{{ }^{G} \lambda_{\mathrm{I}}\left[{ }_{\beta^{-}}^{\beta^{+}}\right](x),{ }^{G} \lambda_{\mathrm{I}}\left[{ }_{\beta^{-}}^{\beta^{+}}\right](y)\right\}=\beta^{+}, \\
\max \left\{{ }^{\mathrm{G}} \lambda_{\mathrm{F}}\left[{ }_{\gamma^{+}}^{\gamma^{-}}\right](x),{ }^{\mathrm{G}} \lambda_{\mathrm{F}}\left[{ }_{\gamma^{+}}^{\gamma^{-}}\right](y)\right\}=\gamma^{-} .
\end{gathered}
$$

Since $G$ is a UP-subalgebra of $X$, we have $x \cdot y \in G$ and so ${ }^{G} \lambda_{T}\left[\alpha_{\alpha^{+}}^{\alpha^{-}}\right](x \cdot y)=\alpha^{-},{ }^{G} \lambda_{I}\left[\beta^{\beta^{+}}\right](x \cdot y)=\beta^{+}$, and ${ }^{\mathrm{G}} \lambda_{\mathrm{F}}\left[_{\gamma^{+}}^{\gamma^{-}}\right](x \cdot y)=\gamma^{-}$. Hence,

$$
\begin{aligned}
& { }^{\mathrm{G}} \lambda_{\mathrm{T}}\left[{ }_{\alpha^{+}}^{\alpha^{-}}\right](x \cdot y)=\alpha^{-} \leqslant \alpha^{-}=\max \left\{{ }^{\mathrm{G}} \lambda_{\mathrm{T}}\left[{ }_{\alpha^{+}}^{\alpha^{-}}\right](x),{ }^{\mathrm{G}} \lambda_{\mathrm{T}}\left[{ }_{\alpha^{+}}^{\alpha^{-}}\right](y)\right\}, \\
& { }^{\mathrm{G}} \lambda_{\mathrm{I}}\left[\begin{array}{l}
\beta^{+} \\
\beta^{-}
\end{array}\right](x \cdot y)=\beta^{+} \geqslant \beta^{+}=\min \left\{{ }^{G} \lambda_{\mathrm{I}}\left[{ }_{\beta^{-}}^{\beta^{+}}\right](x),{ }^{G} \lambda_{\mathrm{I}}\left[{ }_{\beta^{-}}^{\beta^{+}}\right](y)\right\}, \\
& { }^{\mathrm{G}} \lambda_{\mathrm{F}}\left[\left[_{\gamma^{+}}^{\gamma^{-}}\right](x \cdot y)=\gamma^{-} \leqslant \gamma^{-}=\max \left\{{ }^{G} \lambda_{\mathrm{F}} \gamma_{\gamma^{+}}^{\gamma^{-}}\right](x),{ }^{G} \lambda_{\mathrm{F}}\left[{ }_{\gamma^{+}}^{\gamma^{-}}\right](y)\right\} .
\end{aligned}
$$

Case 2: $x \notin \mathrm{G}$ or $\mathrm{y} \notin \mathrm{G}$. Then

$$
\begin{aligned}
& { }^{G} \lambda_{\mathrm{T}}\left[{ }_{\alpha^{-}}^{\alpha^{+}}\right](x)=\alpha^{-} \text {or }{ }^{G} \lambda_{\mathrm{T}}\left[{ }_{\alpha^{-}}^{\alpha^{+}}\right](y)=\alpha^{-}, \\
& { }^{G} \lambda_{I}\left[\begin{array}{c}
\beta^{-} \\
\beta^{+}
\end{array}\right](x)=\beta^{+} \text {or }{ }^{G} \lambda_{I}\left[\begin{array}{c}
\beta^{-} \\
\beta^{+}
\end{array}\right](y)=\beta^{+} \text {, } \\
& { }^{\mathrm{G}} \lambda_{\mathrm{F}}\left[\gamma_{\gamma^{-}}^{\gamma^{+}}\right](\mathrm{x})=\gamma^{-} \text {or }{ }^{\mathrm{G}} \lambda_{\mathrm{F}}\left[\gamma_{\gamma^{-}}^{\gamma^{+}}\right](\mathrm{y})=\gamma^{-} \text {. }
\end{aligned}
$$

Thus

$$
\begin{gathered}
\max \left\{{ }^{G} \lambda_{\mathrm{T}}\left[{ }_{\alpha^{-}}^{\alpha^{+}}\right](x),{ }^{\mathrm{G}} \lambda_{\mathrm{T}}\left[{ }_{\alpha^{-}}^{\alpha^{+}}\right](y)\right\}=\alpha^{-}, \\
\min \left\{{ }^{\mathrm{G}} \lambda_{\mathrm{I}}\left[{ }_{\beta^{+}}^{\beta^{-}}\right](x),{ }^{\mathrm{G}} \lambda_{\mathrm{I}}\left[{ }_{\beta^{+}}^{\beta^{-}}\right](y)\right\}=\beta^{+}, \\
\max \left\{{ }^{\mathrm{G}} \lambda_{\mathrm{F}}\left[{ }_{\gamma^{-}}^{\gamma^{+}}\right](x),{ }^{\mathrm{G}} \lambda_{\mathrm{F}}\left[{ }_{\gamma^{-}}^{\gamma^{+}}\right](y)\right\}=\gamma^{-} .
\end{gathered}
$$

Therefore,

$$
\begin{aligned}
& { }^{\mathrm{G}} \lambda_{\mathrm{T}}\left[\begin{array}{c}
\alpha^{+} \\
\alpha^{-}
\end{array}\right](x \cdot y) \geqslant \alpha^{-}=\max \left\{{ }^{\mathrm{G}} \lambda_{\mathrm{T}}\left[\begin{array}{c}
\alpha^{+} \\
\alpha^{-}
\end{array}\right](x),{ }^{\mathrm{G}} \lambda_{\mathrm{T}}\left[\begin{array}{c}
\alpha^{+} \\
\alpha^{-}
\end{array}\right](y)\right\}, \\
& { }^{G} \lambda_{I}\left[{ }_{\beta^{+}}^{\beta^{-}}\right](x \cdot y) \leqslant \beta^{+}=\min \left\{{ }^{G} \lambda_{I}\left[\beta_{\beta^{+}}^{\beta^{-}}\right](x),{ }^{G} \lambda_{I}\left[\left[_{\beta^{+}}^{\beta^{-}}\right](y)\right\},\right. \\
& { }^{\mathrm{G}} \lambda_{\mathrm{F}}\left[\gamma_{\gamma^{-}}^{\gamma^{+}}\right](x \cdot y) \geqslant \gamma^{-}=\max \left\{{ }^{\mathrm{G}} \lambda_{\mathrm{F}}\left[\gamma_{\gamma^{-}}^{\gamma^{+}}\right](x),{ }^{\mathrm{G}} \lambda_{\mathrm{F}}\left[{ }_{\gamma^{-}}^{\gamma^{+}}\right](y)\right\} \text {. }
\end{aligned}
$$

Hence, $G \wedge\left[\begin{array}{c}\alpha^{+}, \beta^{-}, \gamma^{+} \\ \alpha^{-}, \beta^{+}, \gamma^{-}\end{array}\right]$is a special neutrosophic UP-subalgebra of $X$.

Theorem 2.40. A NS $\mathrm{G}^{\mathrm{N}} \Lambda_{\left[\alpha^{+}, \beta^{-}, \gamma^{+}\right.}^{\alpha^{-}, \beta^{+}}, \gamma^{-}$in $\mathrm{X}$ is a special neutrosophic near UP-filter of $\mathrm{X}$ if and only if a nonempty subset $\mathrm{G}$ of $\mathrm{X}$ is a near UP-filter of $\mathrm{X}$.

Proof. Assume that ${ }^{\mathrm{G}} \wedge\left[\begin{array}{c}\alpha^{-}, \beta^{+}, \gamma^{-} \\ \alpha^{+}, \beta^{-}, \gamma^{+}\end{array}\right]$is a special neutrosophic near UP-filter of X. Since ${ }^{\mathrm{G}} \wedge\left[\begin{array}{c}\left.\alpha^{-}, \beta^{+}, \gamma^{-}\right] \text {satisfies } \\ \alpha^{+}, \beta^{-}, \gamma^{+}\end{array}\right]$satis the condition (2.24), it follows from Lemma 2.38 that $0 \in \mathrm{G}$. Next, let $x \in X$ and $y \in G$. Then ${ }^{G} \lambda_{\mathrm{T}}\left[\alpha_{\alpha^{+}}^{\alpha^{-}}\right](y)=$ $\alpha^{-}$. Thus

$$
{ }^{\mathrm{G}} \lambda_{\mathrm{T}}\left[\begin{array}{l}
\alpha^{-} \\
\alpha^{+}
\end{array}\right](x \cdot y) \leqslant{ }^{G} \lambda_{\mathrm{T}}\left[\begin{array}{l}
\alpha^{-} \\
\alpha^{+}
\end{array}\right](y)=\alpha^{-} \leqslant{ }^{G} \lambda_{\mathrm{T}}\left[\begin{array}{c}
\alpha^{-} \\
\alpha^{+}
\end{array}\right](x \cdot y) \text { by (2.27) }
$$

and so ${ }^{G} \lambda_{\mathrm{T}}\left[\alpha_{\alpha^{+}}^{\alpha^{-}}\right](x \cdot y)=\alpha^{-}$. Thus $x \cdot y \in G$. Hence, $G$ is a near UP-filter of $X$.

Conversely, assume that $G$ is a near UP-filter of $X$. Since $0 \in G$, it follows from Lemma 2.37 that ${ }^{G} \wedge\left[\begin{array}{c}\alpha^{-}, \beta^{+}, \gamma^{-} \\ \alpha^{+}, \beta^{-}, \gamma^{+}\end{array}\right]$satisfies the conditions (2.24), (2.25), and (2.26). Next, let $x, y \in X$. 
Case 1: $y \in G$. Then ${ }^{G} \lambda_{T}\left[\alpha_{\alpha^{+}}^{\alpha^{-}}\right](y)=\alpha^{-},{ }^{G} \lambda_{I}\left[{ }_{\beta^{-}}^{\beta^{+}}\right](y)=\beta^{+}$, and ${ }^{G} \lambda_{F}\left[r_{\gamma^{+}}^{\gamma^{-}}\right](y)=\gamma^{-}$. Since $G$ is a near UP-filter of $X$, we have $x \cdot y \in G$ and so $\left.{ }^{G} \lambda_{T}\left[\alpha_{\alpha^{+}}^{\alpha^{-}}\right](x \cdot y)=\alpha^{-},{ }^{G} \lambda_{I}{ }_{\beta^{-}}^{\beta^{+}}\right](x \cdot y)=\beta^{+}$, and ${ }^{G} \lambda_{F}\left[r_{\gamma^{+}}^{\gamma^{-}}\right](x \cdot y)=\gamma^{-}$. Thus

$$
\begin{aligned}
& { }^{G} \lambda_{\mathrm{T}}\left[\begin{array}{c}
\alpha^{-} \\
\alpha^{+}
\end{array}\right](x \cdot y)=\alpha^{-} \leqslant \alpha^{-}={ }^{G} \lambda_{\mathrm{T}}\left[\begin{array}{c}
\alpha^{+} \\
\alpha^{+}
\end{array}\right](y), \\
& { }^{G} \lambda_{I}\left[\begin{array}{l}
\beta^{+} \\
\beta^{-}
\end{array}\right](x \cdot y)=\beta^{+} \geqslant \beta^{+}={ }^{G} \lambda_{I}\left[\begin{array}{l}
\beta^{+} \\
\beta^{-}
\end{array}\right](y), \\
& { }^{G} \lambda_{F}\left[\begin{array}{l}
\gamma^{-} \\
\gamma^{+}
\end{array}\right](x \cdot y)=\gamma^{-} \leqslant \gamma^{-}={ }^{G} \lambda_{F}\left[{ }_{\gamma^{+}}^{\gamma^{-}}\right](y) .
\end{aligned}
$$

Case 2: $y \notin G$. Then ${ }^{G} \lambda_{T}\left[\begin{array}{c}\alpha^{+} \\ \alpha^{+}\end{array}\right](y)=\alpha^{+},{ }^{G} \lambda_{I}\left[\begin{array}{l}\beta^{+} \\ \beta^{+}\end{array}\right](y)=\beta^{-}$, and ${ }^{G} \lambda_{F}\left[\begin{array}{r}\gamma^{+} \\ \gamma^{-}\end{array}(y)=\gamma^{+}\right.$. Thus

$$
\begin{aligned}
& { }^{G} \lambda_{T}\left[{ }_{\alpha^{+}}^{\alpha^{-}}\right](x \cdot y) \leqslant \alpha^{+}={ }^{G} \lambda_{T}\left[\begin{array}{l}
\alpha^{+} \\
\alpha^{+}
\end{array}\right](y), \\
& { }^{G} \lambda_{I}\left[\begin{array}{l}
\beta^{+} \\
\beta^{-}
\end{array}\right](x \cdot y) \geqslant \beta^{-}={ }^{G} \lambda_{I}\left[\begin{array}{l}
\beta^{+} \\
\beta^{-}
\end{array}\right](y), \\
& { }^{G} \lambda_{F}\left[\begin{array}{l}
\gamma^{+} \\
\gamma^{+}
\end{array}\right](x \cdot y) \leqslant \gamma^{+}={ }^{G} \lambda_{F}\left[\gamma_{\gamma^{+}}^{\gamma^{-}}\right](y) .
\end{aligned}
$$

Hence, ${ }^{G} \wedge\left[\begin{array}{c}\alpha^{-}, \beta^{+}, \gamma^{-} \\ \alpha^{+}, \beta^{-}, \gamma^{+}\end{array}\right]$is a special neutrosophic near UP-filter of $X$.

Theorem 2.41. A NS ${ }^{\mathrm{G}} \Lambda_{\left[\alpha^{+}, \beta^{-}, \gamma^{+}\right.}^{\alpha^{-}, \beta^{+}, \gamma^{-}}$in $\mathrm{X}$ is a special neutrosophic UP-filter of $\mathrm{X}$ if and only if a nonempty subset $\mathrm{G}$ of $\mathrm{X}$ is a UP-filter of $\mathrm{X}$.

Proof. Assume that $G \wedge\left[\begin{array}{c}\alpha^{-}, \beta^{+}, \gamma^{-} \\ \alpha^{+}, \beta^{-}, \gamma^{+}\end{array}\right]$is a special neutrosophic UP-filter of $X$. Since $G \wedge\left[\begin{array}{c}\alpha^{-}, \beta^{+}, \gamma^{-} \\ \alpha^{+}, \beta^{-}, \gamma^{+}\end{array}\right]$satisfies the condition (2.24), it follows from Lemma 2.38 that $0 \in G$. Next, let $x, y \in X$ be such that $x \cdot y \in G$ and $x \in \mathrm{G}$. Then ${ }^{\mathrm{G}} \lambda_{\mathrm{T}}\left[{ }_{\alpha^{+}}^{\alpha^{-}}\right](x \cdot y)=\alpha^{-}={ }^{\mathrm{G}} \lambda_{\mathrm{T}}\left[{ }_{\alpha^{+}}^{\alpha^{-}}\right](x)$. Thus

$$
{ }^{\mathrm{G}} \lambda_{\mathrm{T}}\left[{ }_{\alpha^{+}}^{\alpha^{-}}\right](\mathrm{y}) \leqslant \max \left\{{ }^{\mathrm{G}} \lambda_{\mathrm{T}}\left[{ }_{\alpha^{+}}^{\alpha^{-}}\right](x \cdot y),{ }^{\mathrm{G}} \lambda_{\mathrm{T}}\left[\begin{array}{c}
\alpha_{\alpha^{+}}^{-}
\end{array}\right](x)\right\}=\alpha^{-} \leqslant{ }^{\mathrm{G}} \lambda_{\mathrm{T}}\left[{ }_{\alpha^{+}}^{\alpha^{-}}\right](\mathrm{y}) \text { by }(2.30)
$$

and so ${ }^{G} \lambda_{\mathrm{T}}\left[\begin{array}{c}\alpha^{+} \\ \alpha^{+}\end{array}\right](y)=\alpha^{-}$. Thus $y \in G$. Hence, $G$ is a UP-filter of $X$.

Conversely, assume that $G$ is a UP-filter of $X$. Since $0 \in G$, it follows from Lemma 2.37 that $G \wedge\left[\begin{array}{l}\alpha_{\alpha^{+},}, \beta^{+}, \gamma^{+} \\ \gamma^{-}\end{array}\right]$ satisfies the conditions (2.24), (2.25), and (2.26). Next, let $x, y \in X$.

Case 1: $x \cdot y \in G$ and $x \in G$. Then

$$
\begin{aligned}
& { }^{\mathrm{G}} \lambda_{\mathrm{T}}\left[\begin{array}{c}
\alpha^{-} \\
\alpha^{+}
\end{array}\right](x \cdot y)=\alpha^{-}={ }^{\mathrm{G}} \lambda_{\mathrm{T}}\left[\begin{array}{c}
\alpha^{-} \\
\alpha^{+}
\end{array}\right](x), \\
& { }^{G} \lambda_{I}\left[\begin{array}{l}
\beta^{+} \\
\beta^{-}
\end{array}\right](x \cdot y)=\beta^{+}={ }^{G} \lambda_{I}\left[\begin{array}{l}
\beta^{+} \\
\beta^{-}
\end{array}\right](x), \\
& \left.{ }^{\mathrm{G}} \lambda_{\mathrm{F}}\left[{ }_{\gamma^{+}}^{\gamma^{-}}\right](x \cdot y)=\gamma^{-}={ }^{\mathrm{G}} \lambda_{\mathrm{F}}{ }_{\gamma^{+}}^{\gamma^{-}}\right](x) \text {. }
\end{aligned}
$$

Since $G$ is a UP-filter of $X$, we have $y \in G$ and so ${ }^{G} \lambda_{T}\left[\alpha_{\alpha^{+}}^{\alpha^{-}}\right](y)=\alpha^{-},{ }^{G} \lambda_{I}\left[{ }_{\beta^{-}}^{\beta^{+}}\right](y)=\beta^{+}$, and ${ }^{G} \lambda_{F}\left[\gamma_{\gamma^{+}}^{\gamma^{-}}\right](y)=\gamma^{-}$. Thus

$$
\begin{aligned}
& { }^{G} \lambda_{\mathrm{T}}\left[\alpha_{\alpha^{+}}^{\alpha^{-}}\right](y)=\alpha^{-} \leqslant \alpha^{-}=\max \left\{{ }^{G} \lambda_{\mathrm{T}}\left[\alpha_{\alpha^{+}}^{\alpha^{-}}\right](x \cdot y),{ }^{G} \lambda_{\mathrm{T}}\left[{ }_{\alpha^{+}}^{\alpha^{-}}\right](x)\right\}, \\
& { }^{G} \lambda_{I}\left[\beta_{\beta^{-}}^{\beta^{+}}\right](y)=\beta^{+} \geqslant \beta^{+}=\min \left\{{ }^{G} \lambda_{I}\left[\beta_{\beta^{-}}^{\beta^{+}}\right](x \cdot y),{ }^{G} \lambda_{I}\left[\beta_{\beta^{-}}^{\beta^{+}}\right](x)\right\} \text {, } \\
& \left.\left.{ }^{\mathrm{G}} \lambda_{\mathrm{F}} \gamma_{\gamma^{+}}^{\gamma^{-}}\right](y)=\gamma^{-} \leqslant \gamma^{+}=\max \left\{{ }^{\mathrm{G}} \lambda_{\mathrm{F}} \gamma_{\gamma^{+}}^{\gamma^{-}}\right](x \cdot y),{ }^{\mathrm{G}} \lambda_{\mathrm{F}}\left[\gamma_{\gamma^{+}}^{\gamma^{-}}\right](x)\right\} \text {. }
\end{aligned}
$$

Case 2: $x \cdot y \notin G$ or $x \notin G$. Then

$$
\begin{aligned}
& { }^{\mathrm{G}} \lambda_{\mathrm{T}}\left[{ }_{\alpha^{+}}^{\alpha^{-}}\right](x \cdot y)=\alpha^{+} \text {or }{ }^{\mathrm{G}} \lambda_{\mathrm{T}}\left[{ }_{\alpha^{+}}^{\alpha^{-}}\right](x)=\alpha^{+}, \\
& { }^{\mathrm{G}} \lambda_{\mathrm{I}}\left[\beta_{\beta^{-}}^{\beta^{+}}\right](x \cdot y)=\beta^{-} \text {or }{ }^{\mathrm{G}} \lambda_{\mathrm{I}}\left[{ }_{\beta^{-}}^{\beta^{+}}\right](x)=\beta^{-} \text {, } \\
& \left.{ }^{\mathrm{G}} \lambda_{\mathrm{F}} \mathrm{\gamma}_{\gamma^{+}}^{\gamma^{-}}\right](\mathrm{x} \cdot \mathrm{y})=\gamma^{+} \text {or }{ }^{\mathrm{G}} \lambda_{\mathrm{F}}\left[_{\gamma^{+}}^{\gamma^{-}}\right](\mathrm{x})=\gamma^{+} \text {. }
\end{aligned}
$$


Thus

$$
\begin{gathered}
\max \left\{{ }^{G} \lambda_{\mathrm{T}}\left[{ }_{\alpha^{+}}^{\alpha^{-}}\right](x \cdot y),{ }^{G} \lambda_{\mathrm{T}}\left[\begin{array}{l}
\alpha^{-} \\
\alpha^{+}
\end{array}\right](x)\right\}=\alpha^{+}, \\
\min \left\{{ }^{G} \lambda_{\mathrm{I}}\left[{ }_{\beta^{-}}^{\beta^{+}}\right](x \cdot y),{ }^{G} \lambda_{\mathrm{I}}\left[{ }_{\beta^{-}}^{\beta^{+}}\right](x)\right\}=\beta^{-}, \\
\left.\left.\max ^{{ }^{G}} \lambda_{\mathrm{F}}{ }_{\gamma^{+}}^{\gamma^{-}}\right](x \cdot y),{ }^{G} \lambda_{\mathrm{F}}\left[{ }_{\gamma^{+}}^{\gamma^{-}}\right](x)\right\}=\gamma^{+} .
\end{gathered}
$$

Therefore,

$$
\begin{aligned}
& { }^{G} \lambda_{\mathrm{T}}\left[\begin{array}{c}
\alpha^{-} \\
\alpha^{+}
\end{array}\right](x) \leqslant \alpha^{+}=\max \left\{{ }^{G} \lambda_{T}\left[\begin{array}{c}
\alpha^{-} \\
\alpha^{+}
\end{array}\right](x \cdot y),{ }^{G} \lambda_{T}\left[\begin{array}{c}
\alpha^{-} \\
\alpha^{+}
\end{array}\right](x)\right\}, \\
& { }^{G} \lambda_{I}\left[\beta_{\beta^{-}}^{\beta^{+}}\right](x) \geqslant \beta^{-}=\min \left\{{ }^{G} \lambda_{I}\left[\beta_{\beta^{-}}^{\beta^{+}}\right](x \cdot y),{ }^{G} \lambda_{I}\left[\beta_{\beta^{-}}^{\beta^{+}}\right](x)\right\}, \\
& { }^{\mathrm{G}} \lambda_{\mathrm{F}}\left[\gamma_{\gamma^{+}}^{\gamma^{-}}\right](\mathrm{x}) \leqslant \gamma^{+}=\max \left\{{ }^{\mathrm{G}} \lambda_{\mathrm{F}}\left[{ }_{\gamma^{+}}^{\gamma^{-}}\right](x \cdot y),{ }^{\mathrm{G}} \lambda_{\mathrm{F}}\left[\gamma_{\gamma^{+}}^{\gamma^{-}}\right](x)\right\} \text {. }
\end{aligned}
$$

Hence, $G \wedge\left[\begin{array}{c}\alpha^{-}, \beta^{+}, \gamma^{-} \\ \alpha^{+}, \beta^{-}, \gamma^{+}\end{array}\right]$is a special neutrosophic UP-filter of $X$.

Theorem 2.42. A NS $\mathrm{G}^{\mathrm{N}} \mathcal{L}_{\alpha^{+}, \beta^{-}, \gamma^{+}}^{\alpha^{+}, \beta^{+}, \gamma^{-}}$in $\mathrm{X}$ is a special neutrosophic UP-ideal of $\mathrm{X}$ if and only if a nonempty subset $\mathrm{G}$ of $\mathrm{X}$ is a UP-ideal of $\mathrm{X}$.

Proof. Assume that ${ }^{G} \wedge\left[\begin{array}{c}\alpha^{-}, \beta^{+}, \gamma^{-} \\ \alpha^{+}, \beta^{-}, \gamma^{+}\end{array}\right]$is a special neutrosophic UP-ideal of $X$. Since ${ }^{G} \wedge\left[\begin{array}{c}\alpha^{-}, \beta^{+}, \gamma^{-} \\ \alpha^{+}, \beta^{-}, \gamma^{+}\end{array}\right]$satisfies the condition (2.24), it follows from Lemma 2.38 that $0 \in G$. Next, let $x, y, z \in X$ be such that $x \cdot(y \cdot z) \in G$ and $y \in G$. Then ${ }^{G} \lambda_{\mathrm{T}}\left[{ }_{\alpha^{+}}^{\alpha^{-}}\right](x \cdot(y \cdot z))=\alpha^{-}={ }^{G} \lambda_{T}\left[\begin{array}{c}\alpha^{+} \\ \alpha^{-}\end{array}\right](y)$. Thus

$$
{ }^{\mathrm{G}} \lambda_{\mathrm{T}}\left[\alpha^{\alpha^{+}}\right](x \cdot z) \leqslant \max \left\{{ }^{\mathrm{G}} \lambda_{\mathrm{T}}\left[{ }_{\alpha^{+}}^{\alpha^{-}}\right](x \cdot(y \cdot z)),{ }^{\mathrm{G}} \lambda_{\mathrm{T}}\left[\begin{array}{c}
\alpha^{-} \\
\alpha^{+}
\end{array}\right](y)\right\}=\alpha^{-} \leqslant{ }^{\mathrm{G}} \lambda_{\mathrm{T}}\left[\left[_{\alpha^{+}}^{\alpha^{-}}\right](x \cdot z)\right. \text { by (2.33) }
$$

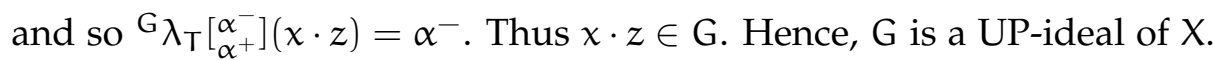

Conversely, assume that $G$ is a UP-ideal of $X$. Since $0 \in G$, it follows from Lemma 2.37 that ${ }^{G} \wedge\left[\begin{array}{c}\alpha^{-}, \beta^{+}, \gamma^{-} \\ \alpha^{+}, \beta^{-}, \gamma^{+}\end{array}\right]$satisfies the conditions (2.24), (2.25), and (2.26). Next, let $x, y, z \in X$.

Case 1: $x \cdot(y \cdot z) \in G$ and $y \in G$. Then

$$
\begin{aligned}
& { }^{\mathrm{G}} \lambda_{\mathrm{T}}\left[\begin{array}{c}
\alpha^{-} \\
\alpha^{+}
\end{array}\right](x \cdot(y \cdot z))=\alpha^{-}={ }^{G} \lambda_{\mathrm{T}}\left[{ }_{\alpha^{+}}^{\alpha^{-}}\right](y), \\
& { }^{{ }^{G}} \lambda_{\mathrm{I}}\left[{ }_{\beta^{-}}^{\beta^{+}}\right](x \cdot(y \cdot z))=\beta^{+}={ }^{G} \lambda_{\mathrm{I}}\left[\begin{array}{l}
\beta^{+} \\
\beta^{-}
\end{array}\right](y), \\
& { }^{{ }^{G}} \lambda_{\mathrm{F}}\left[{ }_{\gamma^{+}}^{\gamma^{-}}\right](x \cdot(y \cdot z))=\gamma-={ }^{G} \lambda_{\mathrm{F}}\left[{ }_{\gamma^{+}}^{\gamma^{-}}\right](y) .
\end{aligned}
$$

Thus

$$
\begin{gathered}
\max \left\{{ }^{\mathrm{G}} \lambda_{\mathrm{T}}\left[{ }_{\alpha^{+}}^{\alpha^{-}}\right](x \cdot(y \cdot z)),{ }^{G} \lambda_{\mathrm{T}}\left[{ }_{\alpha^{+}}^{\alpha^{-}}\right](y)\right\}=\alpha^{-}, \\
\min \left\{{ }^{\mathrm{G}} \lambda_{\mathrm{I}}\left[_{\beta^{-}}^{\beta^{+}}\right](x \cdot(y \cdot z)),{ }^{G} \lambda_{\mathrm{I}}\left[{ }_{\beta^{-}}^{\beta^{+}}\right](y)\right\}=\beta^{+}, \\
\max \left\{{ }^{G} \lambda_{\mathrm{F}}\left[{ }_{\gamma^{+}}^{\gamma^{-}}\right](x \cdot(y \cdot z)),{ }^{G} \lambda_{\mathrm{F}}\left[\gamma_{\gamma^{+}}^{\gamma^{-}}\right](y)\right\}=\gamma^{-} .
\end{gathered}
$$

Since $G$ is a UP-ideal of $X$, we have $x \cdot z \in G$ and so ${ }^{G} \lambda_{T}\left[\alpha_{\alpha^{+}}^{\alpha^{-}}\right](x \cdot z)=\alpha^{-},{ }^{G} \lambda_{I}\left[\beta^{\beta^{+}}\right](x \cdot z)=\beta^{+}$, and ${ }^{\mathrm{G}} \lambda_{\mathrm{F}}\left[\gamma_{\gamma^{+}}^{\gamma^{-}}\right](x \cdot z)=\gamma^{-}$. Thus

$$
\begin{aligned}
& { }^{\mathrm{G}} \lambda_{\mathrm{T}}\left[\begin{array}{l}
\alpha^{-} \\
\alpha^{+}
\end{array}\right](x \cdot z)=\alpha^{-} \leqslant \alpha^{-}=\max \left\{{ }^{\mathrm{G}} \lambda_{\mathrm{T}}\left[\begin{array}{c}
\alpha^{-} \\
\alpha^{+}
\end{array}\right](x \cdot(y \cdot z)),{ }^{\mathrm{G}} \lambda_{\mathrm{T}}\left[\begin{array}{c}
\alpha^{-} \\
\alpha^{+}
\end{array}\right](y)\right\}, \\
& { }^{\mathrm{G}} \lambda_{\mathrm{I}}\left[{ }_{\beta^{-}}^{\beta^{+}}\right](x \cdot z)=\beta^{+} \geqslant \beta^{+}=\min \left\{{ }^{\mathrm{G}} \lambda_{\mathrm{I}}\left[{ }_{\beta^{-}}^{\beta^{+}}\right](x \cdot(y \cdot z)),{ }^{\mathrm{G}} \lambda_{\mathrm{I}}\left[{ }_{\beta^{-}}^{\beta^{+}}\right](y)\right\}, \\
& { }^{\mathrm{G}} \lambda_{\mathrm{F}}\left[{ }_{\gamma^{+}}^{\gamma^{-}}\right](x \cdot z)=\gamma^{-} \leqslant \gamma^{-}=\max \left\{{ }^{\mathrm{G}} \lambda_{\mathrm{F}}\left[\gamma_{\gamma^{+}}^{\gamma^{-}}\right](x \cdot(y \cdot z)),{ }^{\mathrm{G}} \lambda_{\mathrm{F}}\left[{ }_{\gamma^{+}}^{\gamma^{-}}\right](y)\right\} .
\end{aligned}
$$


Case 2: $x \cdot(y \cdot z) \notin G$ or $y \notin G$. Then

$$
\begin{aligned}
& { }^{\mathrm{G}} \lambda_{\mathrm{T}}\left[{ }_{\alpha^{+}}^{\alpha^{-}}\right](x \cdot(y \cdot z))=\alpha^{+} \text {or }{ }^{\mathrm{G}} \lambda_{\mathrm{T}}\left[\begin{array}{c}
\alpha^{+}+ \\
\alpha^{+}
\end{array}\right](y)=\alpha^{+}, \\
& \left.{ }^{G} \lambda_{I}\left[{ }_{\beta^{-}}^{\beta^{+}}\right](x \cdot(y \cdot z))=\beta^{-} \text {or }{ }^{G} \lambda_{I}{ }_{\beta^{-}}^{\beta^{+}}\right](y)=\beta^{-} \text {, } \\
& { }^{\mathrm{G}} \lambda_{\mathrm{F}}\left[_{\gamma^{+}}^{\gamma^{-}}\right](x \cdot(y \cdot z))=\gamma^{+} \text {or }{ }^{\mathrm{G}} \lambda_{\mathrm{F}}\left[\gamma_{\gamma^{+}}^{\gamma^{-}}\right](\mathrm{y})=\gamma^{+} \text {. }
\end{aligned}
$$

Thus

$$
\begin{aligned}
& \max \left\{{ }^{\mathrm{G}} \lambda_{\mathrm{T}}\left[{ }_{\alpha^{+}}^{\alpha^{-}}\right](x \cdot(y \cdot z)),{ }^{\mathrm{G}} \lambda_{\mathrm{T}}\left[{ }_{\alpha^{+}}^{\alpha^{-}}\right](y)\right\}=\alpha^{+}, \\
& \min \left\{{ }^{G} \lambda_{I}\left[\beta_{\beta^{-}}^{\beta^{+}}\right](x \cdot(y \cdot z)),{ }^{G} \lambda_{I}\left[\beta_{\beta^{-}}^{\beta^{+}}\right](y)\right\}=\beta^{-}, \\
& \max \left\{{ }^{\mathrm{G}} \lambda_{\mathrm{F}}\left[{ }_{\gamma^{+}}^{\gamma^{-}}\right](x \cdot(y \cdot z)),{ }^{\mathrm{G}} \lambda_{\mathrm{F}}\left[{ }_{\gamma^{+}}^{\gamma^{-}}\right](y)\right\}=\gamma^{+} \text {. }
\end{aligned}
$$

Therefore,

$$
\begin{aligned}
& { }^{\mathrm{G}} \lambda_{\mathrm{T}}\left[{ }_{\alpha^{+}}^{\alpha^{-}}\right](x \cdot z) \leqslant \alpha^{+}=\max \left\{{ }^{\mathrm{G}} \lambda_{\mathrm{T}}\left[{ }_{\alpha^{+}}^{\alpha^{-}}\right](x \cdot(y \cdot z)),{ }^{\mathrm{G}} \lambda_{\mathrm{T}}\left[\begin{array}{c}
\alpha^{+} \\
\alpha^{+}
\end{array}\right](y)\right\}, \\
& { }^{\mathrm{G}} \lambda_{\mathrm{I}}\left[{ }_{\beta^{-}}^{\beta^{+}}\right](x \cdot z) \geqslant \beta^{-}=\min \left\{{ }^{\mathrm{G}} \lambda_{\mathrm{I}}\left[{ }_{\beta^{-}}^{\beta^{+}}\right](x \cdot(y \cdot z)),{ }^{G} \lambda_{\mathrm{I}}\left[{ }_{\beta^{-}}^{\beta^{+}}\right](y)\right\}, \\
& { }^{\mathrm{G}} \lambda_{\mathrm{F}}\left[{ }_{\gamma^{+}}^{\gamma^{-}}\right](x \cdot z) \leqslant \gamma^{+}=\max \left\{{ }^{G} \lambda_{\mathrm{F}}\left[{ }_{\gamma^{+}}^{\gamma^{-}}\right](x \cdot(y \cdot z)),{ }^{G} \lambda_{\mathrm{F}}\left[{ }_{\gamma^{+}}^{\gamma^{-}}\right](y)\right\} .
\end{aligned}
$$

Hence, $G \wedge\left[\begin{array}{c}\alpha^{-}, \beta^{+}, \gamma^{-} \\ \alpha^{+}, \beta^{-}, \gamma^{+}\end{array}\right]$is a special neutrosophic UP-ideal of $X$.

Theorem 2.43. A NS ${ }^{\mathrm{G}} \wedge_{\left[\alpha^{+}, \beta^{-}, \gamma^{+}\right.}^{\alpha^{-}, \beta^{+}, \gamma^{-}}$in $\mathrm{X}$ is a special neutrosophic strong UP-ideal of $\mathrm{X}$ if and only if a nonempty subset $\mathrm{G}$ of $\mathrm{X}$ is a strong UP-ideal of $\mathrm{X}$.

Proof. Assume that ${ }^{\mathrm{G}} \Lambda_{\left[\alpha^{+}, \beta^{-}, \gamma^{+}\right.}^{\alpha^{-}, \beta^{+}, \gamma^{-}}$is a special neutrosophic strong UP-ideal of $X$. By Theorem 2.28, we have ${ }^{G} \lambda_{\mathrm{T}}\left[\begin{array}{c}\alpha^{-} \\ \alpha^{+}\end{array}\right]$is constant, that is, ${ }^{G} \lambda_{\mathrm{T}}\left[\begin{array}{c}\alpha^{-} \\ \alpha^{+}\end{array}\right]$is constant. Since $\mathrm{G}$ is nonempty, we have ${ }^{G} \lambda_{\mathrm{T}}\left[{ }_{\alpha^{+}}^{\alpha^{-}}\right](x)=\alpha^{-}$for all $x \in X$. Thus $G=X$. Hence, $G$ is a strong UP-ideal of $X$.

Conversely, assume that $G$ is a strong UP-ideal of $X$. Then $G=X$, so

$$
(\forall x \in X)\left(\begin{array}{l}
{ }^{\mathrm{G}} \lambda_{\mathrm{T}}\left[\begin{array}{c}
\alpha^{+} \\
\alpha^{+}
\end{array}\right](x)=\alpha^{-} \\
{ }^{G} \lambda_{\mathrm{I}}\left[\begin{array}{c}
\beta^{+} \\
\beta^{+}
\end{array}\right](x)=\beta^{+} \\
{ }^{G} \lambda_{\mathrm{F}}\left[\begin{array}{c}
\gamma_{\gamma^{+}} \\
{ }^{-}
\end{array}\right](x)=\gamma^{-}
\end{array}\right) .
$$

Thus ${ }^{G} \lambda_{\mathrm{T}}\left[{ }_{\alpha^{+}}^{\alpha^{-}}\right],{ }^{G} \lambda_{I}\left[{ }_{\beta^{-}}^{\beta^{+}}\right]$, and ${ }^{G} \lambda_{F}\left[\gamma_{\gamma^{+}}^{\gamma^{-}}\right]$are constant, that is, $\left.{ }^{G} \Lambda_{\left[\alpha^{+}, \beta^{-}, \gamma^{+}\right.}^{\alpha^{-}, \beta^{+}, \gamma^{-}}\right]$is constant. By Theorem 2.28 , we have ${ }^{G} \wedge\left[\begin{array}{c}\alpha^{-}, \beta^{+}, \gamma^{-} \\ \alpha^{+}, \beta^{-}, \gamma^{+}\end{array}\right]$is a special neutrosophic strong UP-ideal of $X$.

\section{Level subsets of a NS of special types}

In this paper, we discuss the relationships among special neutrosophic UP-subalgebras (resp., special neutrosophic near UP-filters, special neutrosophic UP-filters, special neutrosophic UP-ideals, special neutrosophic strong UP-ideals) of UP-algebras and their level subsets.

Definition 3.1 ([21]). Let $f$ be a fuzzy set in $A$. For any $t \in[0,1]$, the sets

$$
U(f ; t)=\{x \in X \mid f(x) \geqslant t\}, \quad L(f ; t)=\{x \in X \mid f(x) \leqslant t\}, \quad E(f ; t)=\{x \in X \mid f(x)=t\}
$$

are called an upper $\mathrm{t}$-level subset, a lower $\mathrm{t}$-level subset, and an equal $\mathrm{t}$-level subset of $\mathrm{f}$, respectively.

Theorem 3.2. A NS $\Lambda$ in $X$ is a special neutrosophic UP-subalgebra of $X$ if and only if for all $\alpha, \beta, \gamma \in[0,1]$, the sets $\mathrm{L}\left(\lambda_{\mathrm{T}} ; \alpha\right), \mathrm{U}\left(\lambda_{\mathrm{I}} ; \beta\right)$, and $\mathrm{L}\left(\lambda_{\mathrm{F}} ; \gamma\right)$ are UP-subalgebras of $\mathrm{X}$ if $\mathrm{L}\left(\lambda_{\mathrm{T}} ; \alpha\right), \mathrm{U}\left(\lambda_{\mathrm{I}} ; \beta\right)$, and $\mathrm{L}\left(\lambda_{\mathrm{F}} ; \gamma\right)$ are nonempty. 
Proof. Assume that $\Lambda$ is a special neutrosophic UP-subalgebra of $X$. Let $\alpha, \beta, \gamma \in[0,1]$ be such that $\mathrm{L}\left(\lambda_{\mathrm{T}} ; \alpha\right), \mathrm{U}\left(\lambda_{\mathrm{I}} ; \beta\right)$, and $\mathrm{L}\left(\lambda_{\mathrm{F}} ; \gamma\right)$ are nonempty.

Let $x, y \in \mathrm{L}\left(\lambda_{\mathrm{T}} ; \alpha\right)$. Then $\lambda_{\mathrm{T}}(x) \leqslant \alpha$ and $\lambda_{\mathrm{T}}(y) \leqslant \alpha$, so $\alpha$ is a upper bound of $\left\{\lambda_{\mathrm{T}}(x), \lambda_{\mathrm{T}}(y)\right\}$. By (2.21), we have $\lambda_{\mathrm{T}}(x \cdot y) \leqslant \max \left\{\lambda_{\mathrm{T}}(x), \lambda_{\mathrm{T}}(y)\right\} \leqslant \alpha$. Thus $x \cdot y \in \mathrm{L}\left(\lambda_{\mathrm{T}} ; \alpha\right)$.

Let $x, y \in \mathrm{U}\left(\lambda_{\mathrm{I}} ; \beta\right)$. Then $\lambda_{\mathrm{I}}(x) \geqslant \beta$ and $\lambda_{\mathrm{I}}(\mathrm{y}) \geqslant \beta$, so $\beta$ is an lower bound of $\left\{\lambda_{\mathrm{I}}(x), \lambda_{\mathrm{I}}(\mathrm{y})\right\}$. By (2.22), we have $\lambda_{I}(x \cdot y) \geqslant \min \left\{\lambda_{I}(x), \lambda_{I}(y)\right\} \geqslant \beta$. Thus $x \cdot y \in U\left(\lambda_{I} ; \beta\right)$.

Let $x, y \in L\left(\lambda_{F} ; \gamma\right)$. Then $\lambda_{F}(x) \leqslant \gamma$ and $\lambda_{F}(y) \leqslant \gamma$, so $\gamma$ is a upper bound of $\left\{\lambda_{F}(x), \lambda_{F}(y)\right\}$. By (2.23), we have $\lambda_{F}(x \cdot y) \leqslant \max \left\{\lambda_{F}(x), \lambda_{F}(y)\right\} \leqslant \gamma$. Thus $x \cdot y \in L\left(\lambda_{F} ; \gamma\right)$.

Hence, $\mathrm{L}\left(\lambda_{\mathrm{T}} ; \alpha\right), \mathrm{U}\left(\lambda_{\mathrm{I}} ; \beta\right)$, and $\mathrm{L}\left(\lambda_{\mathrm{F}} ; \gamma\right)$ are UP-subalgebras of $X$.

Conversely, assume that for all $\alpha, \beta, \gamma \in[0,1]$, the set $\mathrm{L}\left(\lambda_{\mathrm{T}} ; \alpha\right), \mathrm{U}\left(\lambda_{\mathrm{I}} ; \beta\right)$, and $\mathrm{L}\left(\lambda_{\mathrm{F}} ; \gamma\right)$ are UP-subalgebras if $\mathrm{L}\left(\lambda_{\mathrm{T}} ; \alpha\right), \mathrm{U}\left(\lambda_{\mathrm{I}} ; \beta\right)$, and $\mathrm{L}\left(\lambda_{\mathrm{F}} ; \gamma\right)$ are nonempty.

Let $x, y \in X$. Then $\lambda_{T}(x), \lambda_{T}(y) \in[0,1]$. Choose $\alpha=\max \left\{\lambda_{T}(x), \lambda_{T}(y)\right\}$. Thus $\lambda_{T}(x) \leqslant \alpha$ and $\lambda_{T}(y) \leqslant \alpha$, so $x, y \in \mathrm{L}\left(\lambda_{\mathrm{T}} ; \alpha\right) \neq \emptyset$. By assumption, we have $\mathrm{L}\left(\lambda_{\mathrm{T}} ; \alpha\right)$ is a UP-subalgebra of $\mathrm{X}$ and so $x, y \in \mathrm{L}\left(\lambda_{\mathrm{T}} ; \alpha\right)$. Thus $\lambda_{\mathrm{T}}(x \cdot y) \leqslant \alpha=\max \left\{\lambda_{\mathrm{T}}(x), \lambda_{\mathrm{T}}(\mathrm{y})\right\}$.

Let $x, y \in X$. Then $\lambda_{I}(x), \lambda_{I}(y) \in[0,1]$. Choose $\beta=\min \left\{\lambda_{I}(x), \lambda_{I}(y)\right\}$. Thus $\lambda_{I}(x) \geqslant \beta$ and $\lambda_{I}(y) \geqslant \beta$, so $x, y \in U\left(\lambda_{I} ; \beta\right) \neq \emptyset$. By assumption, we have $U\left(\lambda_{I} ; \beta\right)$ is a UP-subalgebra of $X$ and so $x, y \in U\left(\lambda_{I} ; \beta\right)$. Thus $\lambda_{\mathrm{I}}(x \cdot y) \geqslant \beta=\min \left\{\lambda_{\mathrm{I}}(x), \lambda_{\mathrm{I}}(y)\right\}$.

Let $x, y \in X$. Then $\lambda_{F}(x), \lambda_{F}(y) \in[0,1]$. Choose $\gamma=\max \left\{\lambda_{F}(x), \lambda_{F}(y)\right\}$. Thus $\lambda_{F}(x) \leqslant \gamma$ and $\lambda_{F}(y) \leqslant \gamma$, so $x, y \in \mathrm{L}\left(\lambda_{F} ; \gamma\right) \neq \emptyset$. By assumption, we have $\mathrm{L}\left(\lambda_{F} ; \gamma\right)$ is a UP-subalgebra of $X$ and so $x, y \in \operatorname{L}\left(\lambda_{F} ; \gamma\right)$. Thus $\lambda_{F}(x \cdot y) \leqslant \gamma=\max \left\{\lambda_{F}(x), \lambda_{F}(y)\right\}$.

Therefore, $\Lambda$ is a special neutrosophic UP-subalgebra of $X$.

Theorem 3.3. A NS $\Lambda$ in $X$ is a special neutrosophic near UP-filter of $X$ if and only if for all $\alpha, \beta, \gamma \in[0,1]$, the sets $\mathrm{L}\left(\lambda_{\mathrm{T}} ; \alpha\right), \mathrm{U}\left(\lambda_{\mathrm{I}} ; \beta\right)$, and $\mathrm{L}\left(\lambda_{\mathrm{F}} ; \gamma\right)$ are near $\mathrm{UP}$-filters of $\mathrm{X}$ if $\mathrm{L}\left(\lambda_{\mathrm{T}} ; \alpha\right), \mathrm{U}\left(\lambda_{\mathrm{I}} ; \beta\right)$, and $\mathrm{L}\left(\lambda_{\mathrm{F}} ; \gamma\right)$ are nonempty.

Proof. Assume that $\Lambda$ is a special neutrosophic near UP-filter of $X$. Let $\alpha, \beta, \gamma \in[0,1]$ be such that $\mathrm{L}\left(\lambda_{\mathrm{T}} ; \alpha\right), \mathrm{U}\left(\lambda_{\mathrm{I}} ; \beta\right)$, and $\mathrm{L}\left(\lambda_{\mathrm{F}} ; \gamma\right)$ are nonempty.

Let $x \in \mathrm{L}\left(\lambda_{\mathrm{T}} ; \alpha\right)$. Then $\lambda_{\mathrm{T}}(x) \leqslant \alpha$. By (2.24), we have $\lambda_{\mathrm{T}}(0) \leqslant \lambda_{\mathrm{T}}(x) \leqslant \alpha$. Thus $0 \in \mathrm{L}\left(\lambda_{\mathrm{T}} ; \alpha\right)$. Next, let $y \in \mathrm{L}\left(\lambda_{\mathrm{T}} ; \alpha\right)$. Then $\lambda_{\mathrm{T}}(y) \leqslant \alpha$. By $(2.27)$, we have $\lambda_{\mathrm{T}}(x \cdot y) \leqslant \lambda_{\mathrm{T}}(\mathrm{y}) \leqslant \alpha$. Thus $x \cdot y \in \mathrm{L}\left(\lambda_{\mathrm{T}} ; \alpha\right)$.

Let $x \in U\left(\lambda_{I} ; \beta\right)$. Then $\lambda_{I}(x) \geqslant \beta$. By (2.25), we have $\lambda_{I}(0) \geqslant \lambda_{I}(x) \geqslant \beta$. Thus $0 \in U\left(\lambda_{I} ; \beta\right)$. Next, let $y \in U\left(\lambda_{I} ; \beta\right)$. Then $\lambda_{I}(y) \geqslant \beta$. By $(2.28)$, we have $\lambda_{I}(x \cdot y) \geqslant \lambda_{I}(y) \geqslant \beta$. Thus $x \cdot y \in U\left(\lambda_{I} ; \beta\right)$.

Let $x \in \mathrm{L}\left(\lambda_{F} ; \gamma\right)$. Then $\lambda_{F}(x) \leqslant \gamma$. By (2.26), we have $\lambda_{F}(0) \leqslant \lambda_{F}(x) \leqslant \gamma$. Thus $0 \in \mathrm{L}\left(\lambda_{F} ; \gamma\right)$. Next, $y \in \mathrm{L}\left(\lambda_{F} ; \gamma\right)$. Then $\lambda_{F}(y) \leqslant \gamma$. By $(2.28)$, we have $\lambda_{F}(x \cdot y) \leqslant \lambda_{F}(y) \leqslant \gamma$. Thus $x \cdot y \in L\left(\lambda_{F} ; \gamma\right)$.

Hence, $\mathrm{L}\left(\lambda_{\mathrm{T}} ; \alpha\right), \mathrm{U}\left(\lambda_{\mathrm{I}} ; \beta\right)$, and $\mathrm{L}\left(\lambda_{\mathrm{F}} ; \gamma\right)$ are near UP-filters of $X$.

Conversely, assume that for all $\alpha, \beta, \gamma \in[0,1]$, the set $\mathrm{L}\left(\lambda_{T} ; \alpha\right), \mathrm{U}\left(\lambda_{\mathrm{I}} ; \beta\right)$, and $\mathrm{L}\left(\lambda_{\mathrm{F}} ; \gamma\right)$ are near UP-filters if $\mathrm{L}\left(\lambda_{\mathrm{T}} ; \alpha\right), \mathrm{U}\left(\lambda_{\mathrm{I}} ; \beta\right)$, and $\mathrm{L}\left(\lambda_{\mathrm{F}} ; \gamma\right)$ are nonempty.

Let $x \in X$. Then $\lambda_{T}(0) \in[0,1]$. Choose $\alpha=\lambda_{T}(x)$. Thus $\lambda_{T}(x) \leqslant \alpha$, so $x \in L\left(\lambda_{T} ; \alpha\right) \neq \emptyset$. By assumption, we have $\mathrm{L}\left(\lambda_{T} ; \alpha\right)$ is a near UP-filter of $X$ and so $0 \in \mathrm{L}\left(\lambda_{T} ; \alpha\right)$. Thus $\lambda_{T}(0) \leqslant \alpha=\lambda_{T}(x)$. Next, let $y \in X$. Then $\lambda_{\mathrm{T}}(\mathrm{y}) \in[0,1]$. Choose $\alpha=\lambda_{\mathrm{T}}(\mathrm{y})$. Thus $\lambda_{\mathrm{T}}(\mathrm{y}) \leqslant \alpha$, so $\mathrm{y} \in \mathrm{L}\left(\lambda_{\mathrm{T}} ; \alpha\right) \neq \emptyset$. By assumption, we have $\mathrm{L}\left(\lambda_{\mathrm{T}} ; \alpha\right)$ is a near UP-filter of $X$, and so $x \cdot y \in \mathrm{L}\left(\lambda_{\mathrm{T}} ; \alpha\right)$. Thus $\lambda_{\mathrm{T}}(x \cdot y) \leqslant \alpha=\lambda_{\mathrm{T}}(\mathrm{y})$.

Let $x \in X$. Then $\lambda_{I}(0) \in[0,1]$. Choose $\beta=\lambda_{I}(x)$. Thus $\lambda_{I}(x) \geqslant \beta$, so $x \in U\left(\lambda_{I} ; \beta\right) \neq \emptyset$. By assumption, we have $\mathrm{U}\left(\lambda_{\mathrm{I}} ; \beta\right)$ is a near UP-filter of $X$ and so $0 \in \mathrm{U}\left(\lambda_{\mathrm{I}} ; \beta\right)$. Thus $\lambda_{\mathrm{I}}(0) \geqslant \beta=\lambda_{\mathrm{I}}(x)$. Next, let $y \in X$. Then $\lambda_{\mathrm{I}}(\mathrm{y}) \in[0,1]$. Choose $\beta=\lambda_{\mathrm{I}}(\mathrm{y})$. Thus $\lambda_{\mathrm{I}}(\mathrm{y}) \geqslant \beta$, so $\mathrm{y} \in \mathrm{U}\left(\lambda_{\mathrm{I}} ; \beta\right) \neq \emptyset$. By assumption, we have $\mathrm{U}\left(\lambda_{\mathrm{I}} ; \beta\right)$ is a near UP-filter of $X$, and so $x \cdot y \in U\left(\lambda_{I} ; \beta\right)$. Thus $\lambda_{I}(x \cdot y) \geqslant \beta=\lambda_{I}(y)$.

Let $x \in X$. Then $\lambda_{F}(0) \in[0,1]$. Choose $\gamma=\lambda_{F}(x)$. Thus $\lambda_{F}(x) \leqslant \gamma$, so $x \in L\left(\lambda_{F} ; \gamma\right) \neq \emptyset$. By assumption, we have $L\left(\lambda_{F} ; \gamma\right)$ is a near UP-filter of $X$ and so $0 \in L\left(\lambda_{F} ; \gamma\right)$. Thus $\lambda_{F}(0) \leqslant \gamma=\lambda_{F}(x)$. Next, let $y \in X$. Then $\lambda_{F}(y) \in[0,1]$. Choose $\gamma=\lambda_{F}(y)$. Thus $\lambda_{F}(y) \leqslant \gamma$, so $y \in L\left(\lambda_{F} ; \gamma\right) \neq \emptyset$. By assumption, we have $\mathrm{L}\left(\lambda_{F} ; \gamma\right)$ is a near UP-filter of $X$, and so $x \cdot y \in L\left(\lambda_{F} ; \gamma\right)$. Thus $\lambda_{F}(x \cdot y) \leqslant \gamma=\lambda_{F}(y)$.

Therefore, $\Lambda$ is a special neutrosophic near UP-filter of $X$. 
Theorem 3.4. A NS $\Lambda$ in $X$ is a special neutrosophic UP-filter of $X$ if and only if for all $\alpha, \beta, \gamma \in[0,1]$, the sets $\mathrm{L}\left(\lambda_{\mathrm{T}} ; \alpha\right), \mathrm{U}\left(\lambda_{\mathrm{I}} ; \beta\right)$, and $\mathrm{L}\left(\lambda_{\mathrm{F}} ; \gamma\right)$ are UP-filters of $\mathrm{X}$ if $\mathrm{L}\left(\lambda_{\mathrm{T}} ; \alpha\right), \mathrm{U}\left(\lambda_{\mathrm{I}} ; \beta\right)$, and $\mathrm{L}\left(\lambda_{\mathrm{F}} ; \gamma\right)$ are nonempty.

Proof. Assume that $\Lambda$ is a special neutrosophic UP-filter of $X$. Let $\alpha, \beta, \gamma \in[0,1]$ be such that $L\left(\lambda_{T} ; \alpha\right)$, $\mathrm{U}\left(\lambda_{\mathrm{I}} ; \beta\right)$, and $\mathrm{L}\left(\lambda_{\mathrm{F}} ; \gamma\right)$ are nonempty.

Let $x \in \mathrm{L}\left(\lambda_{\mathrm{T}} ; \alpha\right)$. Then $\lambda_{\mathrm{T}}(x) \leqslant \alpha$. By (2.24), we have $\lambda_{\mathrm{T}}(0) \leqslant \lambda_{\mathrm{T}}(\mathrm{x}) \leqslant \alpha$. Thus $0 \in \mathrm{L}\left(\lambda_{\mathrm{T}} ; \alpha\right)$. Next, let $x \cdot y \in \mathrm{L}\left(\lambda_{T} ; \alpha\right)$ and $x \in \mathrm{L}\left(\lambda_{T} ; \alpha\right)$. Then $\lambda_{T}(x \cdot y) \leqslant \alpha$ and $\lambda_{T}(x) \leqslant \alpha$, so $\alpha$ is a upper bound of $\left\{\lambda_{\mathrm{T}}(x \cdot y), \lambda_{\mathrm{T}}(x)\right\}$. By $(2.30)$, we have $\lambda_{\mathrm{T}}(\mathrm{y}) \leqslant \max \left\{\lambda_{\mathrm{T}}(x \cdot y), \lambda_{\mathrm{T}}(x)\right\} \leqslant \alpha$. Thus $y \in \mathrm{L}\left(\lambda_{\mathrm{T}} ; \alpha\right)$.

Let $x \in U\left(\lambda_{I} ; \beta\right)$. Then $\lambda_{I}(x) \geqslant \beta$. By (2.25), we have $\lambda_{I}(0) \geqslant \lambda_{I}(x) \geqslant \beta$. Thus $0 \in U\left(\lambda_{I} ; \beta\right)$. Next, let $x \cdot y \in U\left(\lambda_{I} ; \beta\right)$ and $x \in U\left(\lambda_{I} ; \beta\right)$. Then $\lambda_{I}(x \cdot y) \geqslant \beta$ and $\lambda_{I}(x) \geqslant \beta$, so $\beta$ is an lower bound of $\left\{\lambda_{I}(x \cdot y), \lambda_{I}(x)\right\}$. By $(2.31)$, we have $\lambda_{I}(y) \geqslant \min \left\{\lambda_{I}(x \cdot y), \lambda_{I}(x)\right\} \geqslant \beta$. Thus $y \in U\left(\lambda_{I} ; \beta\right)$.

Let $x \in \mathrm{L}\left(\lambda_{F} ; \gamma\right)$. Then $\lambda_{F}(x) \leqslant \gamma$. By (2.26), we have $\lambda_{F}(0) \leqslant \lambda_{F}(x) \leqslant \gamma$. Thus $0 \in \mathrm{L}\left(\lambda_{F} ; \gamma\right)$. Next, let $x \cdot y \in \mathrm{L}\left(\lambda_{F} ; \gamma\right)$ and $x \in \mathrm{L}\left(\lambda_{F} ; \gamma\right)$. Then $\lambda_{F}(x \cdot y) \leqslant \gamma$ and $\lambda_{F}(x) \leqslant \gamma$, so $\gamma$ is a upper bound of $\left\{\lambda_{F}(x \cdot y), \lambda_{F}(x)\right\}$. By $(2.32)$, we have $\lambda_{F}(y) \leqslant \max \left\{\lambda_{F}(x \cdot y), \lambda_{F}(x)\right\} \leqslant \gamma$. Thus $y \in L\left(\lambda_{F} ; \gamma\right)$.

Hence, $\mathrm{L}\left(\lambda_{\mathrm{T}} ; \alpha\right), \mathrm{U}\left(\lambda_{\mathrm{I}} ; \beta\right)$, and $\mathrm{L}\left(\lambda_{\mathrm{F}} ; \gamma\right)$ are UP-filters of $X$.

Conversely, assume that for all $\alpha, \beta, \gamma \in[0,1]$, the set $\mathrm{L}\left(\lambda_{T} ; \alpha\right), \mathrm{U}\left(\lambda_{I} ; \beta\right)$, and $\mathrm{L}\left(\lambda_{F} ; \gamma\right)$ are UP-filters if $\mathrm{L}\left(\lambda_{\mathrm{T}} ; \alpha\right), \mathrm{U}\left(\lambda_{\mathrm{I}} ; \beta\right)$, and $\mathrm{L}\left(\lambda_{\mathrm{F}} ; \gamma\right)$ are nonempty.

Let $x \in X$. Then $\lambda_{T}(x) \in[0,1]$. Choose $\alpha=\lambda_{T}(x)$. Thus $\lambda_{T}(x) \leqslant \alpha$, so $x \in L\left(\lambda_{T} ; \alpha\right) \neq \emptyset$. By assumption, we have $\mathrm{L}\left(\lambda_{T} ; \alpha\right)$ is a UP-filter of $X$ and so $0 \in \mathrm{L}\left(\lambda_{T} ; \alpha\right)$. Thus $\lambda_{T}(0) \leqslant \alpha=\lambda_{T}(x)$. Next, let $x, y \in X$. Then $\lambda_{T}(x \cdot y), \lambda_{T}(x) \in[0,1]$. Choose $\alpha=\max \left\{\lambda_{T}(x \cdot y), \lambda_{T}(x)\right\}$. Thus $\lambda_{T}(x \cdot y) \leqslant \alpha$ and $\lambda_{T}(x) \leqslant \alpha$, so $x \cdot y, x \in \mathrm{L}\left(\lambda_{T} ; \alpha\right) \neq \emptyset$. By assumption, we have $\mathrm{L}\left(\lambda_{T} ; \alpha\right)$ is a UP-filter of $X$ and so $y \in \mathrm{L}\left(\lambda_{\mathrm{T}} ; \alpha\right)$. Thus $\lambda_{\mathrm{T}}(\mathrm{y}) \leqslant \alpha=\max \left\{\lambda_{\mathrm{T}}(x \cdot y), \lambda_{\mathrm{T}}(x)\right\}$.

Let $x \in X$. Then $\lambda_{I}(x) \in[0,1]$. Choose $\beta=\lambda_{I}(x)$. Thus $\lambda_{I}(x) \geqslant \beta$, so $x \in U\left(\lambda_{I} ; \beta\right) \neq \emptyset$. By assumption, we have $\mathrm{U}\left(\lambda_{I} ; \beta\right)$ is a UP-filter of $X$ and so $0 \in \mathrm{U}\left(\lambda_{I} ; \beta\right)$. Thus $\lambda_{I}(0) \geqslant \beta=\lambda_{I}(x)$. Next, let $x, y \in X$. Then $\lambda_{I}(x \cdot y), \lambda_{I}(x) \in[0,1]$. Choose $\beta=\min \left\{\lambda_{I}(x \cdot y), \lambda_{I}(x)\right\}$. Thus $\lambda_{I}(x \cdot y) \geqslant \beta$ and $\lambda_{I}(x) \geqslant \beta$, so $x \cdot y, x \in U\left(\lambda_{I} ; \beta\right) \neq \emptyset$. By assumption, we have $\mathrm{U}\left(\lambda_{I} ; \beta\right)$ is a UP-filter of $X$ and so $y \in U\left(\lambda_{I} ; \beta\right)$. Thus $\lambda_{I}(y) \geqslant \beta=\min \left\{\lambda_{I}(x \cdot y), \lambda_{I}(x)\right\}$.

Let $x \in X$. Then $\lambda_{F}(x) \in[0,1]$. Choose $\gamma=\lambda_{F}(x)$. Thus $\lambda_{F}(x) \leqslant \gamma$, so $x \in L\left(\lambda_{F} ; \gamma\right) \neq \emptyset$. By assumption, we have $\mathrm{L}\left(\lambda_{F} ; \gamma\right)$ is a UP-filter of $X$ and so $0 \in \mathrm{L}\left(\lambda_{F} ; \gamma\right)$. Thus $\lambda_{F}(0) \leqslant \gamma=\lambda_{F}(x)$. Next, let $x, y \in X$. Then $\lambda_{F}(x \cdot y), \lambda_{F}(x) \in[0,1]$. Choose $\gamma=\max \left\{\lambda_{F}(x \cdot y), \lambda_{F}(x)\right\}$. Thus $\lambda_{F}(x \cdot y) \leqslant \gamma$ and $\lambda_{F}(x) \leqslant \gamma$, so $x \cdot y, x \in L\left(\lambda_{F} ; \gamma\right) \neq \emptyset$. By assumption, we have $\mathrm{L}\left(\lambda_{F} ; \gamma\right)$ is a UP-filter of $X$ and so $y \in L\left(\lambda_{F} ; \gamma\right)$. Thus $\lambda_{F}(y) \leqslant \gamma=\max \left\{\lambda_{F}(x \cdot y), \lambda_{F}(x)\right\}$.

Therefore, $\Lambda$ is a special neutrosophic UP-filter of $X$.

Theorem 3.5. A NS $\Lambda$ in $X$ is a special neutrosophic UP-ideals of $X$ if and only if for all $\alpha, \beta, \gamma \in[0,1]$, the sets $\mathrm{L}\left(\lambda_{\mathrm{T}} ; \alpha\right), \mathrm{U}\left(\lambda_{\mathrm{I}} ; \beta\right)$, and $\mathrm{L}\left(\lambda_{\mathrm{F}} ; \gamma\right)$ are UP-ideals of $\mathrm{X}$ if $\mathrm{L}\left(\lambda_{\mathrm{T}} ; \alpha\right), \mathrm{U}\left(\lambda_{\mathrm{I}} ; \beta\right)$, and $\mathrm{L}\left(\lambda_{\mathrm{F}} ; \gamma\right)$ are nonempty.

Proof. Assume that $\Lambda$ is a special neutrosophic UP-ideal of $X$. Let $\alpha, \beta, \gamma \in[0,1]$ be such that $L\left(\lambda_{T} ; \alpha\right)$, $\mathrm{U}\left(\lambda_{\mathrm{I}} ; \beta\right)$, and $\mathrm{L}\left(\lambda_{\mathrm{F}} ; \gamma\right)$ are nonempty.

Let $x \in \mathrm{L}\left(\lambda_{\mathrm{T}} ; \alpha\right)$. Then $\lambda_{\mathrm{T}}(x) \leqslant \alpha$. By (2.24), we have $\lambda_{\mathrm{T}}(0) \leqslant \lambda_{\mathrm{T}}(x) \leqslant \alpha$. Thus $0 \in \mathrm{L}\left(\lambda_{\mathrm{T}} ; \alpha\right)$. Next, let $x \cdot(y \cdot z) \in \mathrm{L}\left(\lambda_{\mathrm{T}} ; \alpha\right)$ and $y \in \mathrm{L}\left(\lambda_{\mathrm{T}} ; \alpha\right)$. Then $\lambda_{\mathrm{T}}(x \cdot(y \cdot z)) \leqslant \alpha$ and $\lambda_{\mathrm{T}}(y) \leqslant \alpha$, so $\alpha$ is a upper bound of $\left\{\lambda_{T}(x \cdot(y \cdot z)), \lambda_{T}(y)\right\}$. By (2.33), we have $\lambda_{T}(x \cdot z) \leqslant \max \left\{\lambda_{T}(x \cdot(y \cdot z)), \lambda_{T}(y)\right\} \leqslant \alpha$. Thus $x \cdot z \in L\left(\lambda_{T} ; \alpha\right)$.

Let $x \in \mathrm{U}\left(\lambda_{\mathrm{I}} ; \beta\right)$. Then $\lambda_{\mathrm{I}}(x) \geqslant \beta$. By (2.25), we have $\lambda_{\mathrm{I}}(0) \geqslant \lambda_{\mathrm{I}}(x) \geqslant \beta$. Thus $0 \in \mathrm{U}\left(\lambda_{\mathrm{I}} ; \beta\right)$. Next, let $x \cdot(y \cdot z) \in U\left(\lambda_{I} ; \beta\right)$ and $y \in U\left(\lambda_{I} ; \beta\right)$. Then $\lambda_{I}(x \cdot(y \cdot z)) \geqslant \beta$ and $\lambda_{I}(y) \geqslant \beta$, so $\beta$ is an lower bound of $\left\{\lambda_{I}(x \cdot(y \cdot z)), \lambda_{I}(y)\right\}$. By $(2.34)$, we have $\lambda_{I}(x \cdot z) \geqslant \min \left\{\lambda_{I}(x \cdot(y \cdot z)), \lambda_{I}(y)\right\} \geqslant \beta$. Thus $x \cdot z \in U\left(\lambda_{I} ; \beta\right)$.

Let $x \in \mathrm{L}\left(\lambda_{F} ; \gamma\right)$. Then $\lambda_{F}(x) \leqslant \gamma$. By (2.26), we have $\lambda_{F}(0) \leqslant \lambda_{F}(x) \leqslant \gamma$. Thus $0 \in \mathrm{L}\left(\lambda_{F} ; \gamma\right)$. Next, let $x \cdot(y \cdot z) \in \mathrm{L}\left(\lambda_{F} ; \gamma\right)$ and $y \in \mathrm{L}\left(\lambda_{F} ; \gamma\right)$. Then $\lambda_{F}(x \cdot(y \cdot z)) \leqslant \gamma$ and $\lambda_{F}(y) \leqslant \gamma$, so $\gamma$ is a upper bound of $\left\{\lambda_{F}(x \cdot(y \cdot z)), \lambda_{F}(y)\right\}$. By $(2.35)$, we have $\lambda_{F}(x \cdot z) \leqslant \max \left\{\lambda_{F}(x \cdot(y \cdot z)), \lambda_{F}(y)\right\} \leqslant \gamma$. Thus $x \cdot z \in L\left(\lambda_{F} ; \gamma\right)$.

Hence, $\mathrm{L}\left(\lambda_{T} ; \alpha\right), \mathrm{U}\left(\lambda_{I} ; \beta\right)$, and $\mathrm{L}\left(\lambda_{F} ; \gamma\right)$ are UP-ideals of $X$.

Conversely, assume that for all $\alpha, \beta, \gamma \in[0,1]$, the set $\mathrm{L}\left(\lambda_{\mathrm{T}} ; \alpha\right), \mathrm{U}\left(\lambda_{\mathrm{I}} ; \beta\right)$, and $\mathrm{L}\left(\lambda_{\mathrm{F}} ; \gamma\right)$ are UP-ideals if $\mathrm{L}\left(\lambda_{\mathrm{T}} ; \alpha\right), \mathrm{U}\left(\lambda_{\mathrm{I}} ; \beta\right)$, and $\mathrm{L}\left(\lambda_{\mathrm{F}} ; \gamma\right)$ are nonempty. 
Let $x \in X$. Then $\lambda_{\mathrm{T}}(x) \in[0,1]$. Choose $\alpha=\lambda_{\mathrm{T}}(\mathrm{x})$. Thus $\lambda_{\mathrm{T}}(\mathrm{x}) \leqslant \alpha$, so $x \in \mathrm{L}\left(\lambda_{\mathrm{T}} ; \alpha\right) \neq \emptyset$. By assumption, we have $\mathrm{L}\left(\lambda_{\mathrm{T}} ; \alpha\right)$ is a UP-ideal of $X$ and so $0 \in \mathrm{L}\left(\lambda_{\mathrm{T}} ; \alpha\right)$. Thus $\lambda_{\mathrm{T}}(0) \leqslant \alpha=\lambda_{\mathrm{T}}(x)$. Next, let $x, y, z \in X$. Then $\lambda_{T}(x \cdot(y \cdot z)), \lambda_{T}(y) \in[0,1]$. Choose $\alpha=\max \left\{\lambda_{T}(x \cdot(y \cdot z)), \lambda_{T}(y)\right\}$. Thus $\lambda_{T}(x \cdot(y \cdot z)) \leqslant \alpha$ and $\lambda_{T}(y) \leqslant \alpha$, so $x \cdot(y \cdot z), y \in \mathrm{L}\left(\lambda_{T} ; \alpha\right) \neq \emptyset$. By assumption, we have $\mathrm{L}\left(\lambda_{\mathrm{T}} ; \alpha\right)$ is a UP-ideal of $X$ and so $x \cdot z \in \mathrm{L}\left(\lambda_{\mathrm{T}} ; \alpha\right)$. Thus $\lambda_{\mathrm{T}}(\mathrm{x} \cdot z) \leqslant \alpha=\max \left\{\lambda_{\mathrm{T}}(\mathrm{x} \cdot(\mathrm{y} \cdot z)), \lambda_{\mathrm{T}}(\mathrm{y})\right\}$.

Let $x \in X$. Then $\lambda_{I}(x) \in[0,1]$. Choose $\beta=\lambda_{I}(x)$. Thus $\lambda_{I}(x) \geqslant \beta$, so $x \in U\left(\lambda_{I} ; \beta\right) \neq \emptyset$. By assumption, we have $U\left(\lambda_{I} ; \beta\right)$ is a UP-ideal of $X$ and so $0 \in U\left(\lambda_{I} ; \beta\right)$. Thus $\lambda_{I}(0) \geqslant \beta=\lambda_{I}(x)$. Next, let $x, y, z \in X$. Then $\lambda_{\mathrm{I}}(x \cdot(y \cdot z)), \lambda_{\mathrm{I}}(y) \in[0,1]$. Choose $\beta=\min \left\{\lambda_{\mathrm{I}}(x \cdot(y \cdot z)), \lambda_{\mathrm{I}}(y)\right\}$. Thus $\lambda_{\mathrm{I}}(x \cdot(y \cdot z)) \geqslant \beta$ and $\lambda_{\mathrm{I}}(y) \geqslant \beta$, so $x \cdot(y \cdot z), y \in U\left(\lambda_{I} ; \beta\right) \neq \emptyset$. By assumption, we have $U\left(\lambda_{I} ; \beta\right)$ is a UP-ideal of $X$ and so $x \cdot z \in U\left(\lambda_{I} ; \beta\right)$. Thus $\lambda_{I}(x \cdot z) \geqslant \beta=\min \left\{\lambda_{\mathrm{I}}(x \cdot(y \cdot z)), \lambda_{\mathrm{I}}(y)\right\}$.

Let $x \in X$. Then $\lambda_{F}(x) \in[0,1]$. Choose $\gamma=\lambda_{F}(x)$. Thus $\lambda_{F}(x) \leqslant \gamma$, so $x \in L\left(\lambda_{F} ; \gamma\right) \neq \emptyset$. By assumption, we have $\mathrm{L}\left(\lambda_{\mathrm{F}} ; \gamma\right)$ is a UP-ideal of $X$ and so $0 \in \mathrm{L}\left(\lambda_{\mathrm{F}} ; \gamma\right)$. Thus $\lambda_{F}(0) \leqslant \gamma=\lambda_{F}(x)$. Next, let $x, y, z \in X$. Then $\lambda_{F}(x \cdot(y \cdot z)), \lambda_{F}(y) \in[0,1]$. Choose $\gamma=\max \left\{\lambda_{F}(x \cdot(y \cdot z)), \lambda_{F}(y)\right\}$. Thus $\lambda_{F}(x \cdot(y \cdot z)) \leqslant \gamma$ and $\lambda_{F}(y) \leqslant \gamma$, so $x \cdot(y \cdot z), y \in L\left(\lambda_{F} ; \gamma\right) \neq \emptyset$. By assumption, we have $\mathrm{L}\left(\lambda_{F} ; \gamma\right)$ is a UP-ideal of $X$ and so $x \cdot z \in \mathrm{L}\left(\lambda_{F} ; \gamma\right)$. Thus $\lambda_{\mathrm{F}}(x \cdot z) \leqslant \gamma=\max \left\{\lambda_{\mathrm{F}}(x \cdot(y \cdot z)), \lambda_{\mathrm{F}}(y)\right\}$.

Therefore, $\Lambda$ is a special neutrosophic UP-ideal of $X$.

Definition 3.6 ([23]). Let $\Lambda$ be a NS in X. For $\alpha, \beta, \gamma \in[0,1]$, the sets

$$
\begin{aligned}
\operatorname{uLU}_{\Lambda}(\alpha, \beta, \gamma) & =\left\{x \in X \mid \lambda_{\mathrm{T}} \geqslant \alpha, \lambda_{\mathrm{I}} \leqslant \beta, \lambda_{\mathrm{F}} \geqslant \gamma\right\}, \\
\operatorname{LUL}_{\Lambda}(\alpha, \beta, \gamma) & =\left\{x \in X \mid \lambda_{\mathrm{T}} \leqslant \alpha, \lambda_{\mathrm{I}} \geqslant \beta, \lambda_{\mathrm{F}} \leqslant \gamma\right\}, \\
\mathrm{E}_{\Lambda}(\alpha, \beta, \gamma) & =\left\{x \in X \mid \lambda_{\mathrm{T}}=\alpha, \lambda_{\mathrm{I}}=\beta, \lambda_{\mathrm{F}}=\gamma\right\}
\end{aligned}
$$

are called a ULU- $(\alpha, \beta, \gamma)$-level subset, an LUL- $(\alpha, \beta, \gamma)$-level subset, and an E- $(\alpha, \beta, \gamma)$-level subset of $\Lambda$, respectively. Then we see that

$$
\begin{aligned}
\mathrm{ULU}_{\Lambda}(\alpha, \beta, \gamma) & =\mathrm{U}\left(\lambda_{\mathrm{T}} ; \alpha\right) \cap \mathrm{L}\left(\lambda_{\mathrm{I}} ; \beta\right) \cap \mathrm{U}\left(\lambda_{\mathrm{F}} ; \gamma\right), \\
\mathrm{LUL}_{\Lambda}(\alpha, \beta, \gamma) & =\mathrm{L}\left(\lambda_{\mathrm{T}} ; \alpha\right) \cap \mathrm{U}\left(\lambda_{\mathrm{I}} ; \beta\right) \cap \mathrm{L}\left(\lambda_{\mathrm{F}} ; \gamma\right), \\
\mathrm{E}_{\Lambda}(\alpha, \beta, \gamma) & =\mathrm{E}\left(\lambda_{\mathrm{T}} ; \alpha\right) \cap \mathrm{E}\left(\lambda_{\mathrm{I}} ; \beta\right) \cap \mathrm{E}\left(\lambda_{\mathrm{F}} ; \gamma\right) .
\end{aligned}
$$

Corollary 3.7. A NS $\Lambda$ in $X$ is a special neutrosophic UP-subalgebra of $\mathrm{X}$ if and only if for all $\alpha, \beta, \gamma \in[0,1]$, $\operatorname{LUL}_{\Lambda}(\alpha, \beta, \gamma)$ is a UP-subalgebra of $\mathrm{X}$, where $\operatorname{LUL}_{\Lambda}(\alpha, \beta, \gamma)$ is nonempty.

Proof. It is straightforward by Theorem 3.2.

Corollary 3.8. A NS $\Lambda$ in $X$ is a special neutrosophic near UP-filter of $X$ if and only if for all $\alpha, \beta, \gamma \in[0,1]$, $\operatorname{LUL}_{\Lambda}(\alpha, \beta, \gamma)$ is a near UP-filter of $X$, where $\operatorname{LUL}_{\Lambda}(\alpha, \beta, \gamma)$ is nonempty.

Proof. It is straightforward by Theorem 3.3.

Corollary 3.9. A NS $\wedge$ in $X$ is a special neutrosophic UP-filter of $X$ if and only if for all $\alpha, \beta, \gamma \in[0,1]$, $\operatorname{LUL}_{\Lambda}(\alpha, \beta, \gamma)$ is a UP-filter of $X$, where $\operatorname{LUL}_{\Lambda}(\alpha, \beta, \gamma)$ is nonempty.

Proof. It is straightforward by Theorem 3.4.

Corollary 3.10. A NS $\wedge$ in $X$ is a special neutrosophic UP-ideal of $X$ if and only if for all $\alpha, \beta, \gamma \in[0,1]$, $\operatorname{LUL}_{\Lambda}(\alpha, \beta, \gamma)$ is a UP-ideal of $X$, where $\operatorname{LUL}_{\Lambda}(\alpha, \beta, \gamma)$ is nonempty.

Proof. It is straightforward by Theorem 3.5. 


\section{Conclusions}

In this paper, we have introduced the notions of special neutrosophic UP-subalgebras, special neutrosophic near UP-filters, special neutrosophic UP-filters, special neutrosophic UP-ideals, and special neutrosophic strong UP-ideals of UP-algebras and investigated some of their important properties. Then, we have the diagram of generalization of NSs of special types in UP-algebras as shown in Figure 1.

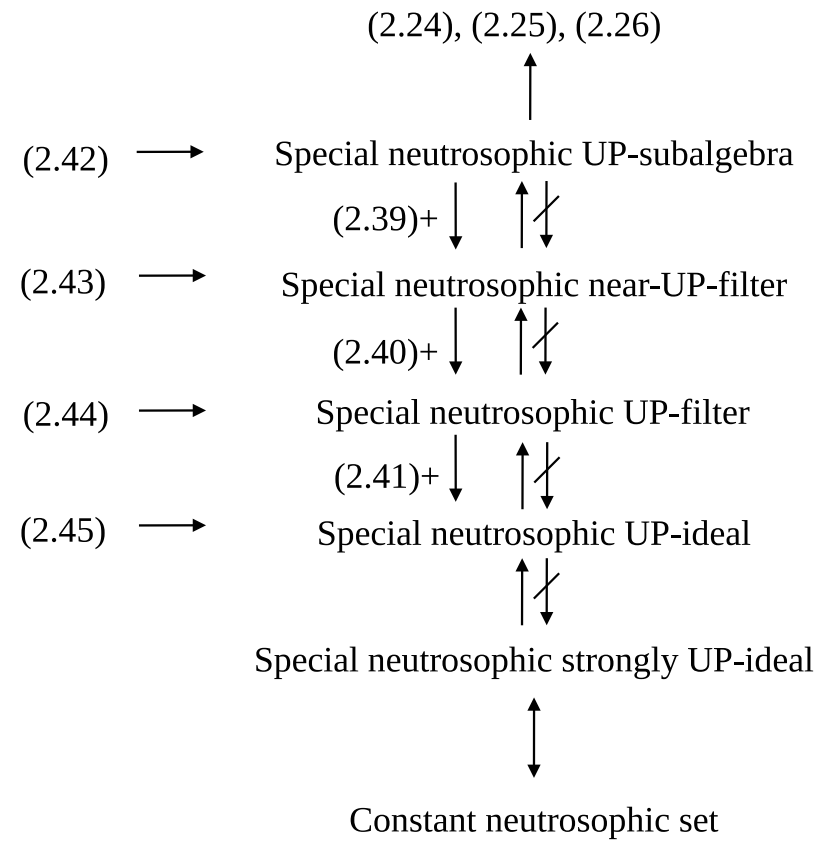

Figure 1: NSs of special types in UP-algebras.

In our future study, we will apply this notion/results to other type of NSs in UP-algebras. Also, we will study the soft set theory/cubic set theory of special neutrosophic UP-subalgebras, special neutrosophic near UP-filters, special neutrosophic UP-filters, special neutrosophic UP-ideals, and special neutrosophic strong UP-ideals.

\section{Acknowledgment}

This work was supported by the Unit of Excellence, University of Phayao. The authors wish to express their sincere thanks to the referees for the valuable suggestions which lead to an improvement of this paper.

\section{References}

[1] M. A. Ansari, A. Haidar, A. N. A. Koam, On a graph associated to UP-algebras, Math. Comput. Appl., 23 (2018), 12 pages. 1

[2] M. A. Ansari, A. N. A. Koam, A. Haider, Rough set theory applied to UP-algebras, Ital. J. Pure Appl. Math., 42 (2019), 388-402. 1

[3] N. Dokkhamdang, A. Kesorn, A. Iampan, Generalized fuzzy sets in UP-algebras, Ann. Fuzzy Math. Inform., 16 (2018), 171-190. 1, 1.2

[4] T. Guntasow, S. Sajak, A. Jomkham, A. Iampan, Fuzzy translations of a fuzzy set in UP-algebras, J. Indones. Math. Soc., 23 (2017), 1-19. 1, 1.4, 1

[5] A. Iampan, A new branch of the logical algebra: UP-algebras, J. Algebra Relat. Topics, 5 (2017), 35-54. 1, 1, 1, 1.4

[6] A. Iampan, Introducing fully UP-semigroups, Discuss. Math. Gen. Algebra Appl., 38 (2018), 297-306. 1

[7] A. Iampan, Multipliers and near UP-filters of UP-algebras, J. Discrete Math. Sci. Cryptography, 2019 (2019), 14 pages. 1.4, 1 
[8] Y. B. Jun, Neutrosophic subalgebras of several types in BCK/BCI-algebras, Ann. Fuzzy Math. Inform., 14 (2017), 75-86. 1

[9] Y. B. Jun, F. Smarandache, H. Bordbar, Neutrosophic N-structures applied to BCK/BCI-algebras, Inform., 8 (2017), 12 pages. 1

[10] Y. B. Jun, F. Smarandache, S. Z. Song, M. Khan, Neutrosophic positive implicative n-ideals in BCK-algebras, Axioms, 7 (2018), 13 pages. 1

[11] W. Kaijae, P. Poungsumpao, S. Arayarangsi, A. Iampan, UP-algebras characterized by their anti-fuzzy UP-ideals and anti-fuzzy UP-subalgebras, Ital. J. Pure Appl. Math., 36 (2016), 667-692. 1

[12] B. Kesorn, K. Maimun, W. Ratbandan, A. Iampan, Intuitionistic fuzzy sets in UP-algebras, Ital. J. Pure Appl. Math., 34 (2015), 339-364. 1

[13] M. Khan, S. Anis, F. Smarandache, Y. B. Jun, Neutrosophic N-structures and their applications in semigroups, Ann. Fuzzy Math. Inform., 14 (2017), 583-598. 1

[14] S. J. Kim, S. Z. Song, Y. B. Jun, Generalizations of neutrosophic subalgebras in BCK/BCI-algebras based on neutrosophic points, Neutrosophic Sets Syst., 20 (2018), 26-35. 1

[15] T. Klinseesook, S. Bukok, A. Iampan, Rough set theory applied to UP-algebras, J. Inf. Optim. Sci., 2019 (2019), 18 pages. 1.3

[16] C. Prabpayak, U. Leerawat, On ideals and congruences in KU-algebras, Sci. Magna, 5 (2009), 54-57. 1

[17] A. Satirad, P. Mosrijai, A. Iampan, Formulas for finding UP-algebras, Int. J. Math. Comput. Sci., 14 (2019), $403-409$. 1

[18] A. Satirad, P. Mosrijai, A. Iampan, Generalized power UP-algebras, Int. J. Math. Comput. Sci., 14 (2019), 17-25. 1.1, 1

[19] F. Smarandache, A unifying field in logics: Neutrosophic logic. neutrosophy, neutrosophic set, neutrosophic probability: Neutrosophic logic: neutrosophy, neutrosophic set, neutrosophic probability, American Research Press, Rehoboth, (1999). $1,2,2.1$

[20] F. Smarandache, Neutrosophic set, a generalization of intuitionistic fuzzy sets, Int. J. Pure Appl. Math., 24 (2005), 287-297. 1

[21] J. Somjanta, N. Thuekaew, P. Kumpeangkeaw, A. Iampan, Fuzzy sets in UP-algebras, Ann. Fuzzy Math. Inform., 12 (2016), 739-756. 1, 1.4, 3.1

[22] M. Songsaeng, A. Iampan, N-fuzzy UP-algebras and its level subsets, J. Algebra Relat. Topics, 6 (2018), 1-24. 1

[23] M. Songsaeng, A. Iampan, Neutrosophic set theory applied to UP-algebras, Eur. J. Pure Appl. Math., 12 (2019), 13821409. 1, 2.2, 2.4, 2.5, 2.6, 2.7, 2.8, 2.9, 2.10, 2.11, 2, 3.6

[24] S. Sripaeng, K. Tanamoon, A. Iampan, On anti Q-fuzzy UP-ideals and anti Q-fuzzy UP-subalgebras of UP-algebras, J. Inf. Optim. Sci., 39 (2018), 1095-1127. 1

[25] M. M. Takallo, H. Bordbar, R. A. Borzooei, Y. B. Jun, BMBJ-neutrosophic ideals in BCK/BCI-algebras, Neutrosophic Sets Syst., 27 (2019), 16 pages. 1

[26] K. Tanamoon, S. Sripaeng, A. Iampan, Q-fuzzy sets in UP-algebras, Songklanakarin J. Sci. Technol., 40 (2018), 9-29. 1

[27] N. Udten, N. Songseang, A. Iampan, Translation and density of a bipolar-valued fuzzy set in UP-algebras, Ital. J. Pure Appl. Math., 41 (2019), 469-496. 2.3

[28] H. Wang, F. Smarandache, Y. Q. Zhang, R. Sunderraman, Interval neutrosophic sets and logic: Theory and applications in computing, Hexis, Phoenix, (2005). 1

[29] L. A. Zadeh, Fuzzy set, Information and Control, 8 (1965), 338-353. 2 